Pacific

Journal of

Mathematics

\title{
ROTA-BAXTER OPERATORS ON THE POLYNOMIAL ALGEBRA, INTEGRATION, AND AVERAGING OPERATORS
}

ShanghuA ZHENG, Li GuO AND MARKus RosenkrANZ

Volume $275 \quad$ No. 2

June 2015 


\title{
ROTA-BAXTER OPERATORS \\ ON THE POLYNOMIAL ALGEBRA, INTEGRATION, AND AVERAGING OPERATORS
}

\author{
Shanghua ZHENG, Li GuO AND MARKus RosenkRANZ
}

The concept of a Rota-Baxter operator is an algebraic abstraction of integration. Following this classical connection, we study the relationship between Rota-Baxter operators and integrals in the case of the polynomial algebra $k[x]$. We consider two classes of Rota-Baxter operators, monomial ones and injective ones. For the first class, we apply averaging operators to determine monomial Rota-Baxter operators. For the second class, we make use of the double product on Rota-Baxter algebras.

1. Introduction 481

2. General concepts and properties 483

3. Monomial Rota-Baxter operators on $\boldsymbol{k}[x]$

4. Injective Rota-Baxter operators on $\boldsymbol{k}[x] \quad 497$

Acknowledgements $\quad 505$

References $\quad 505$

\section{Introduction}

Rota-Baxter operators are deeply rooted in analysis. Their study originated from the work of G. Baxter [1960] on Spitzer's identity [1956] in fluctuation theory. More fundamentally, the notion of Rota-Baxter operator is an algebraic abstraction of the integration by parts formula of calculus. Throughout the 1960s, Rota-Baxter operators were studied by well-known analysts such as Atkinson [1963]. In the 1960s and 1970s, the works of Rota [1969a; 1969b] and Cartier [1972] led the study of Rota-Baxter operators into algebra and combinatorics. In the 1980s, the RotaBaxter operator for Lie algebras was independently discovered by mathematical physicists as the operator form of the classical Yang-Baxter equation [SemenovTian-Shansky 1983]. In the late 1990s, the operator appeared again as a fundamental algebraic structure in the work of Connes and Kreimer [2000] on renormalization of quantum field theory. The present century has witnessed a remarkable renaissance of

MSC2010: primary 16W99; secondary 45N05, 47G10, $12 \mathrm{H} 20$.

Keywords: Rota-Baxter operator, averaging operator, integration, monomial linear operator. 
Rota-Baxter operators through systematic algebraic studies with wide applications to combinatorics, number theory, operads and mathematical physics [Connes and Kreimer 2000; Guo and Keigher 2000; Aguiar 2001; Ebrahimi-Fard et al. 2004; 2006; Bai 2007; Guo and Zhang 2008; Bai et al. 2010; 2013]. See [Guo 2009] for a brief introduction and [Guo 2012] for a more detailed treatment.

Recently, structures related to Rota-Baxter operators, including differential Rota-Baxter algebras [Guo and Keigher 2008] and integro-differential algebras [Rosenkranz and Regensburger 2008], were introduced in the algebraic study of calculus, especially in boundary problems for linear differential equations [Gao et al. 2014; Guo et al. 2014]. The upshot is that the Green's operator of such a boundary problem can be represented by suitable operator rings based on an integro-differential algebra.

In this paper, we revisit the analysis origin of Rota-Baxter operators to study how their algebraic properties are linked with their analytic appearance. We focus on the polynomial algebra $\mathbb{R}[x]$, which plays a central role both in analysis, where it is taken as approximation of analytic functions, and in algebra, where it is the free object in the category of commutative algebras. This algebra, together with the standard integral operator, is also the free commutative Rota-Baxter algebra on the empty set or, in other words, the initial object in the category of commutative Rota-Baxter algebras. Thus it provides an ideal testing ground for the interaction between analytically defined and algebraically defined Rota-Baxter operators.

One natural question in this regard is when an algebraically defined Rota-Baxter operator on $\mathbb{R}[x]$ can be realized in analysis. It is a classical fact that the Riemann integral with variable upper limit is a Rota-Baxter operator of weight zero on $\mathbb{R}[x]$. This remains true when any polynomial is multiplied before the integral operator is applied. We might call these Rota-Baxter operators on $\mathbb{R}[x]$ analytically modeled. It is easy to see that such operators are injective. We conjecture that all injective Rota-Baxter operators on $\mathbb{R}[x]$ are indeed analytically modeled. We provide evidence for this conjecture by exploring two classes of such operators, one class being special but interesting on its own right and the other one being speculatively the general case.

The first comprises what we call monomial Rota-Baxter operators over an arbitrary integral domain $\boldsymbol{k}$ of characteristic zero, meaning Rota-Baxter operators $P$ with $P\left(x^{n}\right)=a x^{k}$, where both $a \in \boldsymbol{k}$ and $k \in \mathbb{N}$ may depend on $n$. We classify monomial Rota-Baxter operators on $\boldsymbol{k}[x]$ and show that all injective monomial Rota-Baxter operators are analytically modeled. The second class is restricted to $\boldsymbol{k}=\mathbb{R}$ and contains those operators that satisfy a differential law $\partial \circ P=r$, where the right-hand side denotes the multiplication operator induced by an arbitrary monomial $r \in \mathbb{R}[x]$. We show that any injective Rota-Baxter operator is of this form and, provided $r$ is monomial, analytically modeled. 
In Section 2, we discuss general algebraic properties of Rota-Baxter operators that will be used in subsequent sections. In Section 3, we focus on monomial RotaBaxter operators. While determining these operators, we prove that all injective monomial Rota-Baxter operators are analytically modeled. In Section 4, we study injective Rota-Baxter operators in general (on the real polynomial ring). We first show that injective Rota-Baxter operators are precisely those that satisfy a differential law. Then we prove that, in the monomial case, they are analytically modeled.

\section{General concepts and properties}

Notation. Let $M$ be a monoid with zero element $0_{M}$. Set $M^{\times}=\left\{x \in M \mid x \neq 0_{M}\right\}$. If $M^{\times}$is closed under the multiplication of $M$, then it is a subsemigroup of $M$. In particular, the monoid of natural numbers (nonnegative integers) is denoted by $\mathbb{N}$, so $\mathbb{N}^{\times}$is the semigroup of positive integers. The notation $l \mid k$ signifies that $l$ is a divisor of $k$.

We use $\boldsymbol{k}$ to denote a commutative ring with identity 1 unless otherwise specified. All $\boldsymbol{k}$-algebras in this paper are assumed to be commutative and with a unit $1_{A}$ that will be identified with $1_{\boldsymbol{k}}$ through the structure map $\boldsymbol{k} \rightarrow A$.

We start by collecting some general properties of Rota-Baxter operators for later use. First we give the definition of a Rota-Baxter $\boldsymbol{k}$-algebra of arbitrary weight:

Definition 2.1 [Baxter 1960; Rota 1995; Guo and Keigher 2000]. Let $\lambda$ be a given element of $\boldsymbol{k}$. A Rota-Baxter $\boldsymbol{k}$-algebra of weight $\lambda$, or simply an RBA of weight $\lambda$, is a pair $(R, P)$ consisting of a $\boldsymbol{k}$-algebra $R$ and a linear operator $P: R \rightarrow R$ that satisfies the Rota-Baxter equation

$$
P(u) P(v)=P(u P(v))+P(P(u) v)+\lambda P(u v) \text { for all } u, v \in R .
$$

In this case $P$ is called a Rota-Baxter operator of weight $\lambda$. If $R$ is only assumed to be a nonunitary $\boldsymbol{k}$-algebra, we call $R$ a nonunitary Rota-Baxter $\boldsymbol{k}$-algebra of weight $\lambda$.

Observe first that the standard integration operator $J_{0}: \boldsymbol{k}[x] \rightarrow \boldsymbol{k}[x]$, given by $x^{n} \mapsto x^{n+1} /(n+1)$, is a (prototypical) Rota-Baxter operator of weight 0 . Of course the choice of initialization point is irrelevant, so for any $a \in \boldsymbol{k}$ there is another weight-0 Rota-Baxter operator $J_{a}: \boldsymbol{k}[x] \rightarrow \boldsymbol{k}[x]$, given by $x^{n} \mapsto$ $\left(x^{n+1}-a^{n+1}\right) /(n+1)$. In this paper we shall only be concerned with the weight- 0 case, so from now on the term "Rota-Baxter operator" is to be understood as "Rota-Baxter operator of weight 0 ".

Recall that from a derivation $\delta$ on a commutative $\boldsymbol{k}$-algebra $R$ one can produce a new derivation $r \delta$ by post-multiplying with any $r \in R$. Analogously, from a Rota-Baxter operator $P$ on $R$ one obtains a new Rota-Baxter operator $P r$ by 
pre-multiplying with any $r \in R$. Indeed,

$$
\begin{aligned}
(P r)(u)(P r)(v) & =P(r u) P(r v)=P(r u P(r v))+P(P(r u) r v) \\
& =(\operatorname{Pr})(u(P r)(v))+(\operatorname{Pr})((\operatorname{Pr})(u) v)
\end{aligned}
$$

for any $u, v \in R$. Applying this to $R=\boldsymbol{k}[x]$, we obtain the family $J_{a} r$ of analytically modeled Rota-Baxter operators on $\boldsymbol{k}[x]$, where $a \in \boldsymbol{k}$ and $r \in \boldsymbol{k}[x]$ are arbitrary. As we will show in Theorem 4.9, in the case of monomials $r$, this family exhausts the injective monomial Rota-Baxter operators.

Let $\operatorname{End}(R):=\operatorname{End}_{\boldsymbol{k}}(R)$ denote the $\boldsymbol{k}$-module of linear operators on $R$. Then the subset $\operatorname{RBO}(R)$ of $\operatorname{End}(R)$ consisting of Rota-Baxter operators $P: R \rightarrow R$ is closed under multiplications by scalars $c \in \boldsymbol{k}$, since in that case $P c=c P$. In the case of derivations on $R$ more is true, since they form a $\boldsymbol{k}$-module (in fact a Lie algebra) while in general the sum of two Rota-Baxter operators is not a Rota-Baxter operator. This motivates the following terminology:

Definition 2.2. (a) We call two Rota-Baxter operators $P_{1}, P_{2} \in \mathrm{RBO}(R)$ compatible if $c_{1} P_{1}+c_{2} P_{2}$ are in $\mathrm{RBO}(R)$ for all $c_{1}, c_{2} \in \boldsymbol{k}$.

(b) Let $P \in \operatorname{RBO}(R)$. Then $Q \in \operatorname{End}(R)$ is called consistent with $P$ if $P-Q$ is in $\operatorname{RBO}(R)$.

(c) For $P, Q \in \operatorname{End}(R)$, we define the bilinear form $\mathrm{RB}(P, Q): R \otimes R \rightarrow R$ by

$$
\mathrm{RB}(P, Q)(u, v):=P(u) Q(v)-P(u Q(v))-Q(P(u) v), \quad u, v \in R .
$$

Thus $P \in \mathrm{RBO}(R)$ means that $\mathrm{RB}(P, P)=0$ on $R \otimes R$.

Recall that for a Rota-Baxter algebra $(R, P)$ the multiplication

$$
\begin{aligned}
\star_{P}: R \otimes R & \rightarrow R, \\
u \star_{P} v & :=P(u) v+u P(v) \text { for all } u, v \in R,
\end{aligned}
$$

is an associative product on $R$, called the double multiplication [Guo 2012, Theorem 1.1.17]. Moreover, $P:(R, \star P) \rightarrow R$ is then a homomorphism of nonunitary Rota-Baxter algebras.

If $A$ is a $\boldsymbol{k}$-module, its (linear) dual is denoted by $A^{*}$. If $A$ is moreover a $\boldsymbol{k}$-algebra, we use the notation

$$
A^{\bullet}:=\left\{\phi \in A^{*} \mid \phi(u v)=\phi(u) \phi(v)\right\}
$$

for the set of multiplicative functionals. Through the structure map $\boldsymbol{k} \rightarrow A$ we may also view the elements of $A^{*}$ as $\boldsymbol{k}$-linear operators from $A$ to $A$, and those of $A^{\bullet}$ as $\boldsymbol{k}$-algebra homomorphisms from $A$ to $\boldsymbol{k}$. 
Proposition 2.3. (a) Two Rota-Baxter operators $P_{1}, P_{2} \in \mathrm{RBO}(R)$ are compatible if and only if $\mathrm{RB}\left(P_{1}, P_{2}\right)+\mathrm{RB}\left(P_{2}, P_{1}\right)=0$. This will be the case in particular when

$$
\begin{aligned}
& P_{1}(u) P_{2}(v)=P_{1}\left(u P_{2}(v)\right)+P_{2}\left(P_{1}(u) v\right), \\
& P_{2}(u) P_{1}(v)=P_{2}\left(u P_{1}(v)\right)+P_{1}\left(P_{2}(u) v\right),
\end{aligned}
$$

hold for all $u, v \in R$.

(b) Let $P \in \operatorname{RBO}(R)$ and $Q \in \operatorname{End}(R)$ be given. Then $Q$ is consistent with $P$ if and only if

$$
\mathrm{RB}(Q, Q)=\mathrm{RB}(P, Q)+\mathrm{RB}(Q, P) .
$$

(c) Let $P$ be in $\operatorname{RBO}(R)$. The set of $f \in R^{*}$ that are consistent with $P$ equals $\left(R, \star_{P}\right)^{\bullet}$.

Proof. (a) For arbitrary $c_{1}, c_{2} \in \boldsymbol{k}$, the bilinear form $\mathrm{RB}\left(c_{1} P_{1}+c_{2} P_{2}, c_{1} P_{1}+c_{2} P_{2}\right)$ is given by

$$
c_{1}^{2} \mathrm{RB}\left(P_{1}, P_{1}\right)+c_{1} c_{2}\left(\mathrm{RB}\left(P_{1}, P_{2}\right)+\mathrm{RB}\left(P_{2}, P_{1}\right)\right)+c_{2}^{2} \mathrm{RB}\left(P_{2}, P_{2}\right),
$$

which simplifies to $c_{1} c_{2}\left(\mathrm{RB}\left(P_{1}, P_{2}\right)+\mathrm{RB}\left(P_{2}, P_{1}\right)\right)$ since $P_{1}, P_{2} \in \operatorname{RBO}(R)$.

(b) Since $P \in \operatorname{RBO}(R)$, we obtain

$$
\mathrm{RB}(P-Q, P-Q)=-\mathrm{RB}(P, Q)-\mathrm{RB}(Q, P)+\mathrm{RB}(Q, Q),
$$

and hence the conclusion.

(c) Using that $P$ is a linear operator and $f$ a linear functional, we have

$$
\begin{gathered}
\operatorname{RB}(f, f)=-f(u) f(v), \\
\operatorname{RB}(f, P)(u, v)=-f(u P(v)), \quad \operatorname{RB}(P, f)(u, v)=-f(P(u) v) .
\end{gathered}
$$

Thus by (b) we conclude that $f$ is consistent with $P$ if and only if

$$
f(u) f(v)=f(P(u) v+u P(v))=f(u \star P v) .
$$

\section{Monomial Rota-Baxter operators on $k[x]$}

In this section, we determine the Rota-Baxter operators on $\boldsymbol{k}[x]$ that send monomials to monomials, called monomial Rota-Baxter operators. We show that the nondegenerate monomial Rota-Baxter operators are analytically modeled. For their independent interest in studying Rota-Baxter operators on $\boldsymbol{k}[x]$, we also consider the degenerate ones. Throughout this section, we assume that $\boldsymbol{k}$ is an integral domain containing $\mathbb{Q}$. 
3A. General properties. We first give general criteria for a monomial linear operator to be a Rota-Baxter operator before specializing in the following sections to the two cases of nondegenerate and degenerate operators.

Definition 3.1. (a) A linear operator $P$ on $\boldsymbol{k}[x]$ is called monomial if for each $n \in \mathbb{N}$

$$
P\left(x^{n}\right)=\beta(n) x^{\theta(n)} \quad \text { with } \beta: \mathbb{N} \rightarrow \boldsymbol{k} \text { and } \theta: \mathbb{N} \rightarrow \mathbb{N} .
$$

If $\beta(n)=0$, the value of $\theta(n)$ does not matter; by convention we set $\theta(n)=0$ in this case.

(b) A monomial operator is called degenerate if $\beta(n)=0$ for some $n \in \mathbb{N}$.

Let $A$ be a nonempty set and let $B$ be a set containing a distinguished element 0 . For a map $\phi: A \rightarrow B$ we define its zero set as $\mathcal{Z}_{\phi}:=\{a \in A \mid \phi(a)=0\}$ and its support as $\mathcal{S}_{\phi}:=A \backslash \mathcal{Z}_{\phi}$. Thus a monomial linear operator $P$ on $\boldsymbol{k}[x]$ is nondegenerate if and only if $\mathcal{Z}_{\beta}=\varnothing$. As the following lemma shows, for a Rota-Baxter operator $P$, degeneracy at $n \in \mathbb{N}$ occurs whenever $P$ is constant on the corresponding monomial:

Lemma 3.2. Let $P$ be a monomial Rota-Baxter operator on $\boldsymbol{k}[x]$ and let $n \in \mathbb{N}$. If $P\left(x^{n}\right)$ is in $\boldsymbol{k}$, then $P\left(x^{n}\right)=0$. In other words, $\mathcal{S}_{\beta}=\mathcal{S}_{\theta}$, and hence $\mathcal{Z}_{\beta}=\mathcal{Z}_{\theta}$.

Proof. If $P\left(x^{n}\right)=c$ is a nonzero constant, then

$$
P\left(x^{n}\right) P\left(x^{n}\right)=c^{2} \neq 2 c^{2}=2 P\left(x^{n} P\left(x^{n}\right)\right) .
$$

Hence $P$ is not a Rota-Baxter operator, and we must have $c=0$.

Theorem 3.3. Let $P$ be a monomial linear operator on $\boldsymbol{k}[x]$ defined by $P\left(x^{n}\right)=$ $\beta(n) x^{\theta(n)}, n \in \mathbb{N}$. Then $P$ is a Rota-Baxter operator if $\theta$ and $\beta$ satisfy the following conditions:

(a) $\mathcal{Z}_{\beta}+\theta\left(\mathcal{S}_{\beta}\right) \subseteq \mathcal{Z}_{\beta}$.

(b) We have

$$
\begin{aligned}
\theta(m)+\theta(n) & =\theta(m+\theta(n))=\theta(\theta(m)+n), \\
\beta(m) \beta(n) & =\beta(m+\theta(n)) \beta(n)+\beta(n+\theta(m)) \beta(m),
\end{aligned}
$$

for all $m, n \in \mathcal{S}_{\beta}$.

Under the assumption that $\mathcal{S}_{\beta}+\theta\left(\mathcal{S}_{\beta}\right) \subseteq \mathcal{S}_{\beta}$, if $P$ is a Rota-Baxter operator then the above conditions hold.

Proof. Since $P$ is a monomial linear operator on $\boldsymbol{k}[x]$, the Rota-Baxter relation in (1) is equivalent to

$$
\begin{aligned}
\beta(m) \beta(n) x^{\theta(m)+\theta(n)}= & \beta(m+\theta(n)) \beta(n) x^{\theta(m+\theta(n))} \\
& +\beta(\theta(m)+n) \beta(m) x^{\theta(\theta(m)+n)} \text { for all } m, n \in \mathbb{N} .
\end{aligned}
$$


Suppose (a) and (b) hold. Since $\mathbb{N}$ is the disjoint union of $\mathcal{Z}_{\beta}$ and $\mathcal{S}_{\beta}$, we can verify (5) by considering the following four cases:

$$
m, n \in \mathcal{Z}_{\beta}, \quad m \in \mathcal{Z}_{\beta}, n \in \mathcal{S}_{\beta}, \quad m \in \mathcal{S}_{\beta}, n \in \mathcal{Z}_{\beta}, \quad m, n \in \mathcal{S}_{\beta} .
$$

In the first case we have $\beta(m)=\beta(n)=0$. Thus (5) holds. In the second case, we have $\beta(m)=0$ and so (5) becomes $\beta(m+\theta(n)) \beta(n)=0$. Then (5) follows from condition (a). The third case can be treated similarly. In the last case, (5) follows from (3) and (4). Thus $P$ is a Rota-Baxter operator on $\boldsymbol{k}[x]$.

Now assume that $\mathcal{S}_{\beta}+\theta\left(\mathcal{S}_{\beta}\right) \subseteq \mathcal{S}_{\beta}$ and that $P$ is a Rota-Baxter operator. Then (5) holds. Taking $m \in \mathcal{Z}_{\beta}$ and $n \in \mathcal{S}_{\beta}$, we obtain $0=\beta(m+\theta(n)) \beta(n) x^{\theta(m+\theta(n))}$. Since $\beta(n) \neq 0$, we must have $\beta(m+\theta(n))=0$, proving (a). Taking $m, n \in \mathcal{S}_{\beta}$, then $\beta(m+\theta(n)) \neq 0$ and $\beta(\theta(m)+n) \neq 0$ by the assumption. Then all the coefficients in (5) are nonzero. Thus the degrees of the monomials must be the same; this yields (3), and (4) follows.

By symmetry, only one of the two identities in (3) is needed. Note also that by definition $A+\varnothing=\varnothing$ for any set $A$, so that $\mathcal{S}_{\beta}+\theta\left(\mathcal{S}_{\beta}\right) \subseteq \mathcal{S}_{\beta}$ and $\mathcal{Z}_{\beta}+\theta\left(\mathcal{S}_{\beta}\right) \subseteq \mathcal{Z}_{\beta}$ are automatic in the nondegenerate case. Otherwise, we obtain the following constraint on $\mathcal{S}_{\beta}$ :

Lemma 3.4. If $P$ is a degenerate monomial Rota-Baxter operator on $\boldsymbol{k}[x]$, then $\mathcal{S}_{\beta}$ is either empty or infinite. The same applies to $\mathcal{Z}_{\beta}$.

Proof. Suppose $\mathcal{S}_{\beta} \neq \varnothing$ and $\left|\mathcal{S}_{\beta}\right|=t<\infty$. Then we may assume that

$$
\mathcal{S}_{\beta}=\left\{m_{i} \in \mathbb{N} \mid 1 \leqslant i \leqslant t, m_{1}<\cdots<m_{t}\right\} .
$$

By (5), $\beta\left(m_{t}\right)^{2}=2 \beta\left(m_{t}\right) \beta\left(m_{t}+\theta\left(m_{t}\right)\right)$. Since $\beta\left(m_{t}\right) \neq 0$, we have $\beta\left(m_{t}\right)=$ $2 \beta\left(m_{t}+\theta\left(m_{t}\right)\right)$, and so $\beta\left(m_{t}+\theta\left(m_{t}\right)\right) \neq 0$. Thus $m_{t}+\theta\left(m_{t}\right)$ is in $\mathcal{S}_{\beta}$. By Lemma 3.2, $\theta\left(m_{t}\right) \geqslant 1$. Then $m_{t}+\theta\left(m_{t}\right)>m_{t}$, a contradiction. Thus either $\mathcal{S}_{\beta}=\varnothing$ or $\left|\mathcal{S}_{\beta}\right|=\infty$.

On the other hand, let $\mathcal{Z}_{\beta} \neq \varnothing$. If $\mathcal{Z}_{\beta}=\mathbb{N}$, then it is certainly infinite. If $\mathcal{Z}_{\beta} \neq \mathbb{N}$, then take $k \in \mathcal{S}_{\beta}$. Since $\mathcal{S}_{\theta}=\mathcal{S}_{\beta}$ by Lemma 3.2, we have $\theta(k)>0$. By Theorem 3.3(a), we obtain $\mathcal{Z}_{\beta}+\theta(k) \subseteq \mathcal{Z}_{\beta}$. This implies that $\mathcal{Z}_{\beta}$ is infinite.

We now give a general setup for constructing monomial Rota-Baxter operators on $\boldsymbol{k}[x]$. This setup will be applied in Section 3B to construct nondegenerate monomial Rota-Baxter operators and in Section $3 \mathrm{C}$ to construct degenerate monomial RotaBaxter operators.

Theorem 3.5. Let $\mathcal{S}$ be a subset of $\mathbb{N}$.

(a) Let the maps $\theta: \mathcal{S} \rightarrow \mathbb{N}^{\times}$and $\beta: \mathcal{S} \rightarrow \boldsymbol{k}^{\times}$satisfy the following conditions:

(i) $\mathbb{N} \backslash \mathcal{S}+\theta(\mathcal{S}) \subseteq \mathbb{N} \backslash \mathcal{S}$.

(ii) Equations (3) and (4) are fulfilled for all $m, n \in \mathcal{S}$. 
Extend $\theta$ and $\beta$ to $\mathbb{N}$ by defining $\theta(n)=0$ and $\beta(n)=0$ for $n \in \mathbb{N} \backslash \mathcal{S}$. Then $P: \boldsymbol{k}[x] \rightarrow \boldsymbol{k}[x]$, defined by $P\left(x^{n}\right)=\beta(n) x^{\theta(n)}$ for $n \in \mathbb{N}$, is a Rota-Baxter operator on $\boldsymbol{k}[x]$.

(b) Let $\theta: \mathcal{S} \rightarrow \mathbb{N}^{\times}$satisfy (3) and $\mathbb{N} \backslash \mathcal{S}+\theta(\mathcal{S}) \subseteq \mathbb{N} \backslash \mathcal{S}$. Extend $\theta$ to $\mathbb{N}$ by defining $\theta(n)=0$ for $n \in \mathbb{N} \backslash \mathcal{S}$. For any $c \in \boldsymbol{k}^{\times}$, define $\beta: \mathbb{N} \rightarrow \boldsymbol{k}$ by

$$
\beta(n)= \begin{cases}c / \theta(n) & n \in \mathcal{S}, \\ 0 & n \notin \mathcal{S} .\end{cases}
$$

Then $P: \boldsymbol{k}[x] \rightarrow \boldsymbol{k}[x]$ defined by $P\left(x^{n}\right)=\beta(n) x^{\theta(n)}$ is a Rota-Baxter operator on $\boldsymbol{k}[x]$.

Proof. (a) This follows from Theorem 3.3.

(b) Under the assumption, for $m, n \in \mathcal{S}$,

$$
\begin{aligned}
\beta(m+\theta(n)) \beta(n)+\beta( & \theta(m)+n) \beta(m) \\
& =\frac{c^{2}}{\theta(m+\theta(n)) \theta(n)}+\frac{c^{2}}{\theta(\theta(m)+n) \theta(m)} \\
& =\frac{c^{2}}{(\theta(m)+\theta(n)) \theta(n)}+\frac{c^{2}}{(\theta(m)+\theta(n)) \theta(m)} \\
& =\frac{c}{\theta(m)} \frac{c}{\theta(n)}=\beta(m) \beta(n)
\end{aligned}
$$

holds. Thus $\theta$ and $\beta$ satisfy the conditions in Theorem 3.3 for $P$ to be a Rota-Baxter operator on $\boldsymbol{k}[x]$.

3B. Nondegenerate case. As mentioned earlier, for a nondegenerate monomial linear operator $P$ on $\boldsymbol{k}[x]$, the conditions $\mathcal{S}_{\beta}+\theta\left(\mathcal{S}_{\beta}\right) \subseteq \mathcal{S}_{\beta}$ and $\mathcal{Z}_{\beta}+\theta\left(\mathcal{S}_{\beta}\right) \subseteq \mathcal{Z}_{\beta}$ are automatic. Thus we obtain a characterization of nondegenerate monomial Rota-Baxter operators from Theorems 3.3 and 3.5:

Corollary 3.6. (a) Let $P$ be a nondegenerate monomial linear operator on $\boldsymbol{k}[x]$ as in (2). Then $P$ is a Rota-Baxter operator if and only if the sequences $\theta$ and $\beta$ satisfy (3) and (4) for all $m, n \in \mathbb{N}$. In this case, $\theta(n) \neq 0$ for all $n \in \mathbb{N}$.

(b) If a sequence $\theta: \mathbb{N} \rightarrow \mathbb{N}$ is nonzero and satisfies (3), then for any $c \in \boldsymbol{k}^{\times}$, the map $\beta: \mathbb{N} \rightarrow \boldsymbol{k}$ given by $\beta(n):=c / \theta(n)$ satisfies (4) and hence gives $a$ Rota-Baxter operator on $\boldsymbol{k}[x]$.

Equation (3) characterizes $\theta$ as an averaging operator defined as follows:

Definition 3.7. (a) A map $\theta: S \rightarrow S$ on a semigroup $S$ is called an averaging operator if

$$
\theta(m \theta(n))=\theta(m) \theta(n)=\theta(\theta(m)+n) \quad \text { for all } m, n \in S .
$$


(b) A linear map $\Theta: R \rightarrow R$ on a $\boldsymbol{k}$-algebra $R$ is called an averaging operator if $\Theta$ is an averaging operator on the multiplicative semigroup of $R$.

The study of averaging operators can be tracked back to Reynolds [1895] and Birkhoff [1950]. We refer the reader to [Pei and Guo 2014] and the references therein for further details.

By Corollary 3.6, a nondegenerate monomial operator $P$ on $\boldsymbol{k}[x]$ is a RotaBaxter operator if and only if the map $\theta$ is an averaging operator on the semigroup $(\mathbb{N},+)$, and the corresponding $\boldsymbol{k}$-linear operator $\Theta: x^{n} \mapsto x^{\theta(n)}$ makes $(\boldsymbol{k}[x], \Theta)$ into an averaging algebra. We write $\mathcal{A}$ for the set of all nondegenerate averaging operators, i.e., sequences $\theta: \mathbb{N} \rightarrow \mathbb{N}^{\times}$satisfying (3). We describe $\mathcal{A}$ as the first step to determine nondegenerate monomial Rota-Baxter operators on $\boldsymbol{k}[x]$. We denote the free semigroup over $\mathbb{N}^{\times}$by $S\left(\mathbb{N}^{\times}\right)$, so the elements $\sigma \in S\left(\mathbb{N}^{\times}\right)$are finite sequences $\left(\sigma_{0}, \ldots, \sigma_{d-1}\right)$ of positive numbers having any length $d>0$.

Theorem 3.8. There is a bijective correspondence $\Phi: \mathcal{A} \rightarrow S\left(\mathbb{N}^{\times}\right)$given by

$$
\Phi(\theta)=(\theta(0), \ldots, \theta(d-1)) / d
$$

with

$$
d:=\min \left\{j \in \mathbb{N}^{\times} \mid \theta(r+j)=\theta(r)+j \text { for all } r \in \mathbb{N}\right\},
$$

whose inverse maps $\sigma:=\left(\sigma_{0}, \ldots, \sigma_{d-1}\right) \in S\left(\mathbb{N}^{\times}\right)$to the map $\theta: \mathbb{N} \rightarrow \mathbb{N}^{\times}$defined by $\theta(n)=\left(\ell+\sigma_{j}\right) d$ for $n=\ell d+j$ with $\ell \in \mathbb{N}$ and $0 \leqslant j<d$. Moreover, we have $\operatorname{im} \theta=d \mathbb{N}_{\geqslant s}$ for $s:=\min (\sigma)$.

Proof. First consider $\theta \in \mathcal{A}$. Defining the map $\tilde{\theta}:=\theta-\mathrm{id}_{\mathbb{N}}: \mathbb{N} \rightarrow \mathbb{Z}$, one obtains from (3) that $\tilde{\theta}(m+\theta(n))=\tilde{\theta}(m)$ for all $m, n \in \mathbb{N}$. Hence $\tilde{\theta}$ is periodic, and $d$ is well-defined as the primitive period of $\tilde{\theta}$. Since every $\theta(n)$ is also a period of $\tilde{\theta}$, this implies $\operatorname{im} \theta \subseteq d \mathbb{N}^{\times}$so that the given map $\Phi: \mathcal{A} \rightarrow S\left(\mathbb{N}^{\times}\right)$is well-defined.

Next let us write $\Psi$ for the assignment $\sigma \mapsto \theta$ defined above. By checking (3) one sees that this yields a well-defined map $\Psi: S\left(\mathbb{N}^{\times}\right) \rightarrow \mathcal{A}$.

Now we prove $\Phi \circ \Psi=\operatorname{id}_{S\left(\mathbb{N}^{\times}\right)}$. So let $\theta: \mathbb{N} \rightarrow \mathbb{N}^{\times}$be the map defined as above by a given sequence $\left(\sigma_{0}, \ldots, \sigma_{d-1}\right) \in S\left(\mathbb{N}^{\times}\right)$. Since $\tilde{\theta}(n)=\sigma_{j} d-j$ for $n=\ell d+j$, we see that $d$ is a period of the map $\tilde{\theta}$. Assume $d$ is greater than its primitive period $d^{\prime}$. Then $d=k d^{\prime}$ for $k>1$, and

$$
\sigma_{0} k d^{\prime}=\theta(0)=\tilde{\theta}(0)=\tilde{\theta}\left(d^{\prime}\right)=\sigma_{d^{\prime}} d-d^{\prime}=\left(\sigma_{d^{\prime}} k-1\right) d^{\prime}
$$

implies $\sigma_{0} k=\sigma_{d^{\prime}} k-1$, which contradicts $k>1$. We conclude that $d$ is the primitive period of $\tilde{\theta}$, so the definition of $\Phi$ recovers the correct value of $d$. Moreover, for $j=0, \ldots, d-1$ we have $\theta(j)=\sigma_{j} d$, which implies $\Phi(\theta)=\sigma$ as required. 
It remains to prove the converse relation $\Psi \circ \Phi=\mathrm{id}_{\mathcal{A}}$. Taking an arbitrary $\theta \in \mathcal{A}$, we must prove that it coincides with the sequence $\theta^{\prime}$ defined by $\theta^{\prime}(\ell d+j)=$ $(\ell+\theta(j) / d) d=\ell d+\theta(j)$ for any $\ell \in \mathbb{N}$ and $0 \leqslant j<d$. For these values we must then show that $\theta(\ell d+j)=\ell d+\theta(j)$, which is equivalent to $\tilde{\theta}(\ell d+j)=\tilde{\theta}(j)$. The latter is ensured since we know that $\tilde{\theta}$ has primitive period $d$.

As noted above, $\operatorname{im} \theta \subseteq d \mathbb{N}^{\times}$so $\theta / d: \mathbb{N} \rightarrow \mathbb{N}^{\times}$is well-defined. We must show $\operatorname{im}(\theta / d)=\mathbb{N}_{\geqslant s}$. The inclusion from left to right follows since $(\theta / d)(\ell d+j)=$ $\ell+\sigma_{j} \geqslant \sigma_{j} \geqslant s$. Now let $n \geqslant s$ be given and write $s=\sigma_{j}$ for some $j=0, \ldots, d-1$. Then $\ell:=n-\sigma_{j} \in \mathbb{N}$ is such that $(\theta / d)(\ell d+j)=n$, which establishes the inclusion from right to left.

As sequences, the relation between $\theta: \mathbb{N} \rightarrow \mathbb{N}^{\times}$and $\sigma:\{0, \ldots, d-1\} \rightarrow \mathbb{N}^{\times}$ can be written as $\theta / d=(\sigma, \sigma+1, \sigma+2, \ldots)$, where $1,2, \ldots$ designate constant sequences of length $d$. More precisely, we have

$$
\theta / d=\left(\sigma_{0}, \ldots, \sigma_{d-1}, \sigma_{0}+1, \ldots, \sigma_{d-1}+1, \ldots\right) .
$$

Theorem 3.8 yields a construction algorithm for the map $\theta$ from a nondegenerate monomial Rota-Baxter operator:

Algorithm 3.9. Every sequence $\theta: \mathbb{N} \rightarrow \mathbb{N}$ corresponding to a nondegenerate monomial Rota-Baxter operator on $\boldsymbol{k}[x]$ can be generated as follows:

(a) Let $d \in \mathbb{N}^{\times}$be given. For each $j=0, \ldots, d-1$, fix $\sigma_{j} \in \mathbb{N}^{\times}$.

(b) For $n \in \mathbb{N}$ with $n=\ell d+\bar{n}$, where $\bar{n} \in\{0, \ldots, d-1\}$ is the remainder of $n$ modulo $d$, define

$$
\theta(n):=n+\sigma_{\bar{n}} d-\bar{n}=\ell d+\sigma_{\bar{n}} d .
$$

We consider two extreme cases of Algorithm 3.9 of particular interest:

Case 1 . If $d=1$ one can only choose $\theta(0) \neq 0$ so that $\theta(n)=n+\theta(0)$ for all $n \in \mathbb{N}$.

Case 2. For $d>1$ and $\sigma_{j}=1,0 \leqslant j \leqslant d-1$, we obtain $\theta(n)=n+d-\bar{n}=(\ell+1) d$ with $n=\ell d+\bar{n}$.

Example 3.10. Setting $d=2$ and $\sigma_{0}=\sigma_{1}=1$, we choose the sequence $\beta$ according to Corollary 3.6(b) with $c=2$. Then the $\boldsymbol{k}$-linear map $P: \boldsymbol{k}[x] \rightarrow \boldsymbol{k}[x]$ defined by

$$
P\left(x^{2 k}\right)=\frac{x^{2 k+2}}{k+1} \quad \text { and } \quad P\left(x^{2 k+1}\right)=\frac{x^{2 k+2}}{k+1}
$$

is a nondegenerate Rota-Baxter operator on $\boldsymbol{k}[x]$.

Next we determine all $\beta$ for the sequences $\theta$ coming from the two extreme cases above. 
Theorem 3.11. (a) Let $d=1$ with $\theta(n)=n+k$ for some $k \in \mathbb{N}^{\times}$. Then $\beta: \mathbb{N} \rightarrow \boldsymbol{k}$ satisfies (4) if and only if $\beta(n)=c / \theta(n)$ for some $c \in \boldsymbol{k}^{\times}$.

(b) Let $d>1$ be given with $\theta(n)=n+d-\bar{n}$. Then $\beta: \mathbb{N} \rightarrow \boldsymbol{k}$ satisfies (4) if and only if it is defined as follows: Fix $c_{j} \in \boldsymbol{k}^{\times}$and assign $\beta(j):=1 / c_{j}$ for $0 \leqslant j \leqslant d-1$. Then for any $n \in \mathbb{N}$ with $n=\ell d+\bar{n}$ define $\beta(n)=\beta(\bar{n}) /(\ell+1)$.

Proof. (a) For a $\theta$ of the given form, by (4), we have

$$
\beta(n) \beta(0)=\beta(n+k)(\beta(0)+\beta(n)) .
$$

Set $\beta(0):=a$ for some $a \in \boldsymbol{k}^{\times}$and write $c:=k a$. Then $\beta(0)=c / k$ and $c$ is in $\boldsymbol{k}^{\times}$. We next prove $\beta(n)=c /(n+k)$ by induction on $n \geqslant 0$. The base case $n=0$ is true. Assume $\beta(n)=c /(n+k)$ has been proved for $n \geqslant 0$. By (7), we obtain

$$
\beta(n+1-k) \beta(0)=\beta(n+1)(\beta(0)+\beta(n+1-k)) .
$$

Since $k \geqslant 1$, we obtain $n+1-k \leqslant n$. By the induction hypothesis, we get $\beta(n+1-k)=c /(n+1)$. Then by (8) we have

$$
\beta(n+1)=\frac{c^{2} /(k(n+1))}{c / k+c /(n+1)}=\frac{c}{n+1+k} .
$$

This completes the induction. Thus $\beta(n)=c / \theta(n)$ for some $c \in \boldsymbol{k}^{\times}$and all $n \in \mathbb{N}$. The converse follows from Corollary 3.6(b).

(b) Taking $\gamma(n)=1 / \beta(n)$, (4) is equivalent to

$$
\frac{\gamma(m)}{\gamma(m+\theta(n))}+\frac{\gamma(n)}{\gamma(\theta(m)+n)}=1 .
$$

Thus we just need to show that, for a fixed sequence $\theta$ in the theorem, a sequence $\gamma: \mathbb{N} \rightarrow \boldsymbol{k}$ satisfies (9) if and only if $\gamma$ is defined by $\gamma(n)=(\ell+1) \gamma(\bar{n})$ if $n=\ell d+\bar{n}$, where the $\gamma(\bar{n}) \in \boldsymbol{k}^{\times}$for $\bar{n} \in\{0, \ldots, d-1\}$ are arbitrarily preassigned.

$(\Rightarrow)$ Take $m=0$ and $n=\ell d$ with $\ell \geqslant 0$ in (9). After simplifying we obtain

$$
\gamma((\ell+1) d)=\gamma(\ell d)+\gamma(0) .
$$

Then by an induction on $\ell$, we obtain

$$
\gamma(\ell d)=(\ell+1) \gamma(0) \text {. }
$$

Next note that, for $n=\ell d+\bar{n}$,

$$
\theta(n)=\ell d+d
$$

holds. Then for $j \in\{0, \ldots, d-1\}$, taking $m=0$ and $n=\ell d+j$ in (9) we obtain

$$
1=\frac{\gamma(0)}{\gamma(\theta(\ell d+j))}+\frac{\gamma(\ell d+j)}{\gamma(\theta(0)+\ell d+j)}=\frac{\gamma(0)}{\gamma(\ell d+d)}+\frac{\gamma(\ell d+j)}{\gamma(d+\ell d+j)} \text {. }
$$


This gives

$$
\gamma((\ell+1) d+j)=\frac{\ell+2}{\ell+1} \gamma(\ell d+j),
$$

and recursively yields

$$
\gamma(\ell d+j)=(\ell+1) \gamma(j) .
$$

$(\Leftarrow)$ Conversely, suppose a sequence $\beta$ is given by $\gamma(n)=(\ell+1) \gamma(\bar{n})$ if $n=\ell d+\bar{n}$, for preassigned $\gamma(\bar{n})$ as specified above. Then for any $m, n \in \mathbb{N}$ with $m=k d+\bar{m}$ and $n=\ell d+\bar{n}$, by (11) we obtain

$$
\begin{aligned}
\frac{\gamma(m)}{\gamma(m+\theta(n))}+ & \frac{\gamma(n)}{\gamma(\theta(m)+n)} \\
& =\frac{\gamma(k d+\bar{m})}{\gamma(k d+\bar{m}+\theta(\ell d+\bar{n}))}+\frac{\gamma(\ell d+\bar{n})}{\gamma(\theta(k d+\bar{m})+\ell d+\bar{n})} \\
& =\frac{\gamma(k d+\bar{m})}{\gamma(k d+\bar{m}+\ell d+d)}+\frac{\gamma(\ell d+\bar{n})}{\gamma(k d+d+\ell d+\bar{n})} \\
& =\frac{(k+1) \gamma(\bar{m})}{(k+\ell+2) \gamma(\bar{m})}+\frac{(\ell+1) \gamma(\bar{n})}{(k+\ell+2) \gamma(\bar{n})}=1 .
\end{aligned}
$$

This is (9).

In the special case of polynomial sequences $\theta: \mathbb{N} \rightarrow \mathbb{N}$ and $\alpha=1 / \beta: \mathbb{N} \rightarrow \boldsymbol{k}$, the range of possibilities can be drastically narrowed down.

Theorem 3.12. Suppose $\boldsymbol{k}$ is a field containing $\mathbb{Q}$. Let $P: \boldsymbol{k}[x] \rightarrow \boldsymbol{k}[x]$ be a nondegenerate monomial linear operator with $P\left(x^{n}\right)=(1 / \alpha(n)) x^{\theta(n)}$ for $n \in \mathbb{N}$, and assume $\theta(n)$ as well as $\alpha(n)$ are polynomials. Then $P$ is a Rota-Baxter operator if and only if

$$
\theta(n)=n+k \quad \text { and } \quad \alpha(n)=c(n+k),
$$

for some $k \in \mathbb{N}^{\times}$and some $c \in \boldsymbol{k}^{\times}$.

Proof. By Corollary 3.6, the operator $P$ defined by (12) is a Rota-Baxter operator. So we just need to show that any Rota-Baxter operator given by (2) with polynomial sequences $\theta(n)$ and $\alpha(n)$ must satisfy the conditions in (12). Since $P$ is a RotaBaxter operator, (3) gives the characteristic relation $2 \theta(n)=\theta(\theta(n)+n)$. But $\theta$ and $\alpha$ are polynomials with $\operatorname{deg} \theta$ and $\operatorname{deg} \alpha$ respectively. Checking degrees, let us first assume $\operatorname{deg} \theta \geqslant 2$. In this case we have

$$
\operatorname{deg} 2 \theta=\operatorname{deg} \theta<(\operatorname{deg} \theta)^{2}=\operatorname{deg} \theta(\theta(n)+n),
$$

which contradicts the characteristic relation. Thus, $\operatorname{deg} \theta \leqslant 1$, and we can write $\theta(n)=s n+k$ for some $s, k \in \mathbb{N}$. The characteristic relation becomes $2(s n+k)=$ $s(s n+n+k)+k$, or equivalently $(s n+k)(s-1)=0$. If $s \neq 1$, we obtain 
$s n+k=0$ for all $n \in \mathbb{N}$. But then $s=k=0$, and $P$ is the zero operator, which contradicts the hypothesis that $P$ is nondegenerate. Therefore $s=1$ and hence $\theta(n)=n+k$ as claimed in (12).

For deriving the second condition of (12), we specialize (4) to obtain $2 \alpha(n)=$ $\alpha(\theta(n)+n)$ and hence the recursion $2 \alpha(n)=\alpha(2 n+k)$. Set $\ell=\operatorname{deg} \alpha$ and suppose the leading coefficient of $\alpha$ is $c \in \boldsymbol{k}^{\times}$. Now taking leading coefficients of the recursion, we get $2 c=2^{\ell} c$ and thus $\ell=1$. This means we can write $\alpha(n)=c n+c_{0}$ for some $c \in \boldsymbol{k}^{\times}$and $c_{0} \in \boldsymbol{k}$. Substituting this into the recursion leads to $2\left(c n+c_{0}\right)=c(2 n+k)+c_{0}$ and hence $\alpha(n)=c(n+k)$ as claimed in (12). It remains to show that $k \neq 0$. But this follows because $P(1)=x^{k} / c k$ so that necessarily $c k \neq 0$.

Next we investigate injective monomial Rota-Baxter operators and show them to be analytically modeled. We note first that if $P$ is degenerate, then there exists $n_{0} \in \mathbb{N}$ such that $\beta\left(n_{0}\right)=0$, and then $P\left(x^{n_{0}}\right)=0$. Thus ker $P \neq\{0\}$ and $P$ is not injective. Thus any injective monomial Rota-Baxter operator is nondegenerate.

Theorem 3.13. Let $P$ be a monomial Rota-Baxter operator on $\boldsymbol{k}[x]$. The following statements are equivalent:

(a) The operator $P$ is injective.

(b) The $\theta$ in (2) from $P$ satisfies $\theta(n)=n+k$ for some $k \in \mathbb{N}^{\times}$.

(c) There are $k \in \mathbb{N}^{\times}$and $c \in \boldsymbol{k}^{\times}$such that $P\left(x^{n}\right)=c \int_{0}^{x} t^{n+k-1} d t$ and hence $P=c J_{0} x^{k-1}$.

Proof. (a) $\Rightarrow$ (b) Assume that $P$ is an injective monomial Rota-Baxter operator. Then $P$ is nondegenerate. By Algorithm 3.9, there are $d \geqslant 1$ and $\sigma_{j} \in \mathbb{N}^{\times}$for $j \in\{0, \ldots, d-1\}$ such that $\theta(n)=\ell d+\sigma_{\bar{n}} d$, where $n=\ell d+\bar{n}$ and $\bar{n}$ is the remainder of $n$ modulo $d$. Suppose $d>1$. Without loss of generality, we may assume $\sigma_{0} \geqslant \sigma_{1}$ so that $n:=\left(\sigma_{0}-\sigma_{1}\right) d+1>0$. Since $\theta(0)=\sigma_{0} d$, we obtain

$$
\theta(n)=\theta\left(\left(\sigma_{0}-\sigma_{1}\right) d+1\right)=\left(\sigma_{0}-\sigma_{1}\right) d+\sigma_{1} d=\theta(0),
$$

hence $\theta$ is not injective. This forces $d=1$. Then, by the first case considered after Algorithm 3.9, we have $\theta(n)=n+k$ for fixed $k \geqslant 1$.

(b) $\Rightarrow$ (c) For a $\theta$ of the given form, by Theorem 3.11(a), we have $\beta(n)=c / \theta(n)$ for some $c \in \boldsymbol{k}^{\times}$. Thus

$$
P\left(x^{n}\right)=\beta(n) x^{\theta(n)}=\frac{c}{n+k} x^{n+k}=c \int_{0}^{x} t^{n+k-1} d t,
$$

as needed.

(c) $\Rightarrow$ (a) Since $P\left(x^{n}\right)=c \int_{0}^{x} t^{n+k-1} d t=(c /(n+k)) x^{n+k}$ for all $n \in \mathbb{N}$, the
operator $P$ is injective. 
3C. Degenerate case. We next apply Theorem 3.5 to construct degenerate monomial Rota-Baxter operators on $\boldsymbol{k}[x]$ when $\mathcal{S}_{\beta}$ is either $k \mathbb{N}$, where $k \geqslant 1$, or $\mathbb{N} \backslash(k \mathbb{N})$, where $k \geqslant 2$.

Proposition 3.14. Let $P\left(x^{n}\right)=\beta(n) x^{\theta(n)}, n \in \mathbb{N}$ and define a monomial linear operator on $\boldsymbol{k}[x]$ such that $\mathcal{S}_{\beta}=k \mathbb{N}$ for some $k>0$. Then $P$ is a Rota-Baxter operator on $\boldsymbol{k}[x]$ if and only if $\theta(k m)=\tilde{\theta}(m) \in \mathcal{S}_{\beta}{ }^{\times}$and $\beta(k m)=\tilde{\beta}(m), m \geqslant 0$ for maps $\tilde{\theta}: \mathbb{N} \rightarrow \mathbb{N}$ and $\tilde{\beta}: \mathbb{N} \rightarrow \boldsymbol{k}$ that satisfy

$$
\tilde{\theta}\left(m_{1}\right)+\tilde{\theta}\left(m_{2}\right)=\tilde{\theta}\left(m_{1}+\frac{1}{k} \tilde{\theta}\left(m_{2}\right)\right)=\tilde{\theta}\left(\frac{1}{k} \tilde{\theta}\left(m_{1}\right)+m_{2}\right)
$$

and

$$
\tilde{\beta}\left(m_{1}\right) \tilde{\beta}\left(m_{2}\right)=\tilde{\beta}\left(m_{1}+\frac{1}{k} \tilde{\theta}\left(m_{2}\right)\right) \tilde{\beta}\left(m_{2}\right)+\tilde{\beta}\left(m_{2}+\frac{1}{k} \tilde{\theta}\left(m_{1}\right)\right) \tilde{\beta}\left(m_{1}\right)
$$

for all $m_{1}, m_{2} \in \mathbb{N}$.

Proof. Since $\mathcal{S}_{\beta}=\{k m \mid m \in \mathbb{N}\}, \mathcal{Z}_{\beta}=\{k m+i \mid 1 \leqslant i \leqslant k-1, m \in \mathbb{N}\}$. Suppose $P$ is a Rota-Baxter operator on $\boldsymbol{k}[x]$. Then by (1), we have $P\left(x^{k m_{1}+i} P\left(x^{k m_{2}}\right)\right)=0$ for all $m_{1}, m_{2} \in \mathbb{N}$ and $1 \leqslant i \leqslant k-1$. Thus $\beta\left(k m_{2}\right) \beta\left(k m_{1}+i+\theta\left(k m_{2}\right)\right)=0$. Since $\beta\left(k m_{2}\right) \neq 0$, we obtain $\beta\left(k m_{1}+i+\theta\left(k m_{2}\right)\right)=0$. Then $k m_{1}+i+\theta\left(k m_{2}\right)$ is in $\mathcal{Z}_{\beta}$, and then $i+\theta\left(k m_{2}\right)$ is in $\mathcal{Z}_{\beta}$ for $1 \leqslant i \leqslant k-1$. Suppose that there exists $m_{0} \in \mathbb{N}$ such that $\theta\left(k m_{0}\right) \not \equiv 0(\bmod k)$. Then there exists $1 \leqslant i_{0} \leqslant k-1$ such that $i_{0}+\theta\left(k m_{0}\right) \equiv 0(\bmod k)$. So $i_{0}+\theta\left(k m_{0}\right)$ is in $\mathcal{S}_{\beta}$ by the definition of $\mathcal{S}_{\beta}$. This is a contradiction to the fact proved above that $i+\theta\left(k m_{2}\right)$ is in $\mathcal{Z}_{\beta}$ for $1 \leqslant i \leqslant k-1$.

Thus $\theta(\mathrm{km})$ is in $\mathcal{S}_{\beta}{ }^{\times}$for all $m \in \mathbb{N}$. So $k n+\theta(\mathrm{km})$ is in $\mathcal{S}_{\beta}$ for all $n, m \in \mathbb{N}$. By Theorem 3.3, equations (3) and (4) hold. Let $\tilde{\theta}(\mathrm{m}):=\theta(\mathrm{km})$ and let $\tilde{\beta}(\mathrm{m}):=$ $\beta(k m), m \in \mathbb{N}$. Thus $\tilde{\beta}(m) \neq 0$ for all $m \in \mathbb{N}$. Then by (3) and (4), equations (13) and (14) hold. This is what we want. The converse follows from Theorem 3.5(a).

Proposition 3.14 gives a large class of monomial Rota-Baxter operators on $\boldsymbol{k}[x]$ with $\mathcal{S}_{\beta}=k \mathbb{N}$, reducing to Corollary 3.6 for $k=1$. On the other hand, Theorem 3.5 also gives the following result on monomial Rota-Baxter operators on $\boldsymbol{k}[x]$, where $\mathcal{S}_{\beta}$ is now complementary to Proposition 3.14:

Proposition 3.15. Let $P\left(x^{n}\right)=\beta(n) x^{\theta(n)}$ be a monomial linear operator on $\boldsymbol{k}[x]$ with $\mathcal{S}_{\beta}=\mathbb{N} \backslash k \mathbb{N}$ for some $k \geqslant 2$.

(a) For any $t \in \mathbb{N}^{\times}$one obtains a degenerate monomial $R B O$ by setting $\theta(k m+i)=$ $k(m+t)$ and $\theta(\mathrm{km})=0$ for $m \in \mathbb{N}$ and $1 \leqslant i \leqslant k-1$, choosing $\beta$ as in Theorem 3.5(b).

(b) Assume that $\theta(i)=k$ for $1 \leqslant i \leqslant k-1$. Then $\theta$ corresponds to a degenerate monomial $R B O$ on $\boldsymbol{k}[x]$ if and only if $\theta(k m+i)=k(m+1)$ for all $m \in \mathbb{N}$ and $1 \leqslant i \leqslant k-1$. 
Proof. (a) By our assumption on $\mathcal{S}_{\beta}, \mathcal{Z}_{\beta}=\{\mathrm{km} \mid m \in \mathbb{N}\}$. By assumption $\theta(k m+i)=k(m+t)$ for all $m \in \mathbb{N}$ and $1 \leqslant i \leqslant k-1$, hence we obtain $\mathcal{Z}_{\beta}+\theta\left(\mathcal{S}_{\beta}\right) \subseteq$ $\mathcal{Z}_{\beta}$. Since

$$
\begin{gathered}
\theta\left(k m_{1}+i_{1}\right)+\theta\left(k m_{2}+i_{2}\right)=k\left(m_{1}+m_{2}+2 t\right), \\
\theta\left(k m_{1}+i_{1}+\theta\left(k m_{2}+i_{2}\right)\right)=\theta\left(k\left(m_{1}+m_{2}+t\right)+i_{1}\right)=k\left(m_{1}+m_{2}+2 t\right),
\end{gathered}
$$

for all $m_{1}, m_{2} \in \mathbb{N}$ and $1 \leqslant i_{1}, i_{2} \leqslant k-1$, we obtain (3). Thus we may apply Theorem 3.5(b) to obtain a degenerate RBO $P$ on $\boldsymbol{k}[x]$.

(b) Assume first that $P$ is a monomial RBO on $\boldsymbol{k}[x]$. Then by (5), for all $m \in \mathbb{N}$ and $1 \leqslant i \leqslant k-1$, we obtain $\beta(k m+i) \beta(k m+\theta(k m+i))=0$. Since $\beta(k m+i) \neq 0$, we have $\beta(k m+\theta(k m+i))=0$, so $k m+\theta(k m+i)$ is in $\mathcal{Z}_{\beta}$. From $\mathcal{Z}_{\beta}=k \mathbb{N}$ we infer $\theta(k m+i) \in \mathcal{Z}_{\beta}$. Thus $\mathcal{S}_{\beta}+\theta\left(\mathcal{S}_{\beta}\right) \subseteq \mathcal{S}_{\beta}$. By Theorem 3.3, (3) holds. We now prove that $\theta(k m+i)=k(m+1)$ by induction on $m \geqslant 0$. The base case $m=0$ is immediate from our assumption. Assume that $\theta(k m+i)=k(m+1)$ has been proved for $m \geqslant 0$. By (3), we have

$$
\theta(k(m+1)+i)=\theta(k m+i+\theta(i))=\theta(k m+i)+\theta(i) .
$$

By the induction hypothesis, we get $\theta(k(m+1)+i)=k(m+2)$. This completes the proof.

Conversely, by $\theta(k m+i)=k(m+1)$ and (a), we obtain a degenerate RBO $P$ on $\boldsymbol{k}[x]$.

Example 3.16. Taking $k=2$ in Propositions 3.14 and 3.15, we obtain the following degenerate monomial Rota-Baxter operators on $\boldsymbol{k}[x]$ :

(a) $P\left(x^{2 k}\right)=x^{2(k+1)} /(k+1)$ and $P\left(x^{2 k+1}\right)=0$ for all $k \in \mathbb{N}$.

(b) $P\left(x^{2 k}\right)=0$ and $P\left(x^{2 k+1}\right)=x^{2(k+1)} /(k+1)$ for all $k \in \mathbb{N}$.

The above examples may also be regarded as special cases of the following result:

Proposition 3.17. Let $P_{0} \in \mathrm{RBO}(R)$ for a $\boldsymbol{k}$-algebra $R$. Assume that $\phi$ is a $\boldsymbol{k}$ linear operator on $R$ such that $E:=P_{0}(\mathrm{im} \phi)$ is a nonunitary $\boldsymbol{k}$-subalgebra. If $\phi$ is a homomorphism of the E-module $R$, then $P_{0} \circ \phi$ is also a Rota-Baxter operator on $R$.

Proof. This follows immediately since

$$
\begin{aligned}
\left(P_{0} \circ \phi\right)(a)\left(P_{0} \circ \phi\right)(b) & =P_{0}(\phi(a)) P_{0}(\phi(b)) \\
& =P_{0}\left(\phi(a) P_{0}(\phi(b))\right)+P_{0}\left(P_{0}(\phi(a)) \phi(b)\right) \\
& =\left(P_{0} \circ \phi\right)\left(a\left(P_{0} \circ \phi\right)(b)\right)+\left(P_{0} \circ \phi\right)\left(\left(P_{0} \circ \phi\right)(a) b\right)
\end{aligned}
$$

for all $a, b \in R$. 
For $R=\boldsymbol{k}[x]$, let $\phi: f(x) \mapsto \frac{1}{2}(f(x)+f(-x))$ be the projector onto the $\boldsymbol{k}$-subspace spanned by the even monomials, and set $P_{0}=2 J_{0} x$. Then

$$
\left(P_{0} \circ \phi\right)\left(x^{n}\right):= \begin{cases}x^{2(k+1)} /(k+1) & \text { if } n=2 k, \\ 0 & \text { if } n=2 k+1,\end{cases}
$$

for all $k \in \mathbb{N}$ so that $P_{0} \circ \phi$ is the same as $P$ in Example 3.16(a). On the other hand, choosing $\phi$ as the projector $f(x) \mapsto \frac{1}{2}(f(x)-f(-x))$ onto the space of odd monomials and setting $P_{0}=2 J_{0}$ yields

$$
\left(P_{0} \circ \phi\right)\left(x^{n}\right):= \begin{cases}0 & \text { if } n=2 k, \\ x^{2(k+1)} /(k+1) & \text { if } n=2 k+1,\end{cases}
$$

for all $k \in \mathbb{N}$ so that $P_{0} \circ \phi$ is the same as $P$ in Example 3.16(b). In both cases, $E$ is the nonunitary algebra of nonconstant even monomials.

Proposition 3.18. Let $P\left(x^{n}\right)=\beta(n) x^{\theta(n)}$ be a nonzero degenerate monomial $R B O$ on $\boldsymbol{k}[x]$ satisfying the condition $\mathcal{S}_{\beta}+\theta\left(\mathcal{S}_{\beta}\right) \subseteq \mathcal{S}_{\beta}$.

(a) There exists a map $\sigma: \mathbb{N} \rightarrow \mathcal{S}_{\beta}$ such that $P_{0}\left(x^{n}\right):=P\left(x^{\sigma(n)}\right)$ defines a nondegenerate monomial $R B O$ on $\boldsymbol{k}[x]$.

(b) We have the disjoint union

$$
\mathcal{S}_{\beta}=C \uplus\left(s_{1}+e \mathbb{N}\right) \uplus \cdots \uplus\left(s_{k}+e \mathbb{N}\right),
$$

where $C \subset \mathbb{N}$ is finite, $k<e \in \mathbb{N}^{\times}$, and $s_{1}, \ldots, s_{k} \in \mathcal{S}_{\beta}$ are incongruent modulo e (in the sense that $x-y \notin e \mathbb{Z}$ ) such that $s_{1}-e, \ldots, s_{k}-e \notin C$. Moreover, there exists a finite set $E \subset \mathcal{S}_{\beta}$ such that $\theta$ is determined uniquely by its values on $E$.

Proof. Since $P$ is nonzero, both $\mathcal{S}_{\beta} \neq \varnothing$ and $\mathcal{Z}_{\beta} \neq \varnothing$ are infinite by Lemma 3.4. From (3) and the condition $\mathcal{S}_{\beta}+\theta\left(\mathcal{S}_{\beta}\right) \subseteq \mathcal{S}_{\beta}$ we see that $T:=\theta\left(\mathcal{S}_{\beta}\right)$ is additively closed. As in the proof of Theorem 3.8, one checks that $\theta-\mathrm{id}_{\mathbb{N}}$ is periodic on $\mathcal{S}_{\beta}$ with primitive period $d$ and $T \subseteq d \mathbb{N}^{\times}$so that $d \mid e:=\operatorname{gcd}(T)$. Hence $T / e$ is a numerical semigroup [Rosales and García-Sánchez 1999, Proposition 10.1], meaning a subsemigroup of $\mathbb{N}^{\times}$with a finite complement $G \subseteq \mathbb{N}^{\times}$of so-called gaps. Thus we obtain $T=e \mathbb{N}^{\times} \backslash e G$. We write $f \in \mathbb{N}$ for the Frobenius number of $T / e$, meaning the greatest element of $G$ for $G \neq \varnothing$ and $f=0$ otherwise.

(a) Fix an element $s$ of $\mathcal{S}_{\beta}$. We define $\sigma: \mathbb{N} \rightarrow \mathcal{S}_{\beta}$ as follows. For $n \in \mathbb{N}$, write $n=\ell e+r$ with $\ell \geqslant 0$ and $0 \leqslant r<e$. Define $\sigma(\ell e+r):=(f+\ell+1) e+s$. Then $\sigma: \mathbb{N} \rightarrow \mathcal{S}_{\beta}$ follows from the condition $\mathcal{S}_{\beta}+T \subseteq \mathcal{S}_{\beta}$ since $(f+\ell+1) e \in T$ for all $\ell \geqslant 0$. We show now that

$$
\sigma(n+\theta(\sigma(m)))=\sigma(n)+\theta(\sigma(m))
$$


for all $m, n \in \mathbb{N}$. We have $\theta(\sigma(m))=t e \in T$ for some $t \notin G$, and we may write $n=\ell e+r$ with $0 \leqslant r<e$ and $\ell \geqslant 0$. Then one computes $\sigma(r)+(\ell+t) e$ for both sides of (16).

Let us now prove that $P_{0}$ satisfies (1), or equivalently $\mathrm{RB}\left(P_{0}, P_{0}\right)=0$. Since the latter is a symmetric bilinear form and $\boldsymbol{k}[x]$ has characteristic zero, the polarization identity implies that it suffices to prove $\operatorname{RB}\left(P_{0}, P_{0}\right)(u, u)=0$ for all $u \in \boldsymbol{k}[x]$. Of course we may restrict ourselves to the canonical basis $u=x^{n}$, so it remains to show $P\left(x^{\sigma(n)}\right)^{2}=2 P_{0}\left(x^{n} P\left(x^{\sigma(n)}\right)\right)$. Applying the definition of $P$, this is equivalent to

$$
\beta(\sigma(n))^{2} x^{2 \theta(\sigma(n))}=2 \beta(\sigma(n)) P_{0}\left(x^{n+\theta(\sigma(n))}\right),
$$

and we may use (16) to expand the right-hand side further to

$$
2 \beta(\sigma(n)) \beta(\sigma(n)+\theta(\sigma(n))) x^{\theta(\sigma(n)+\theta(\sigma(n)))} .
$$

But now we may apply Equations (3) and (4) from Theorem 3.3 to conclude that this is equal to the left-hand side. Hence $P_{0}$ is indeed a monomial RBO on $\boldsymbol{k}[x]$. Clearly $P_{0}\left(x^{n}\right)=P\left(x^{\sigma(n)}\right) \neq 0$ since $\sigma(n) \in \mathcal{S}_{\beta}$, so $P_{0}$ is nondegenerate.

(b) For defining $s_{1}, \ldots, s_{k}$, consider first the sets $\Sigma_{i}:=\mathcal{S}_{\beta} \cap(i+e \mathbb{N})$ for $i \in$ $\{0, \ldots, e-1\}$. Suppressing the empty ones, we reindex the rest as $\Sigma_{1}, \ldots, \Sigma_{k}$ for $1 \leqslant k \leqslant e$. Then for any $i \in\{1, \ldots, k\}$ there exists $\sigma_{i} \in \Sigma_{i}$ such that $\sigma_{i}+e \mathbb{N} \subseteq \Sigma_{i}$. Indeed, one may choose $\sigma_{i}=\sigma_{i}^{\prime}+(f+1) e$ for any $\sigma_{i}^{\prime} \in \Sigma_{i}$ since then $(f+1) e \in T$, and the hypothesis $\mathcal{S}_{\beta}+T \subseteq \mathcal{S}_{\beta}$ implies the required condition $\sigma_{i}+e \mathbb{N} \subseteq \Sigma_{i}$. Let $s_{i} \in \Sigma_{i}$ be minimal such that the condition is satisfied; this implies in particular $s_{i}-e \notin \mathcal{S}_{\beta}$. Then clearly $\Sigma_{i}=C_{i} \uplus\left(s_{i}+e \mathbb{N}\right)$ for finite sets $C_{i} \subset \mathbb{N}$. Now define $C:=C_{1} \cup \cdots \cup C_{k}$ to obtain the decomposition (15). We must have $k<e$ since otherwise $\mathcal{Z}_{\beta} \subseteq\left\{0, \ldots, \max \left(s_{1}, \ldots, s_{e}\right)\right\}$ is finite, contradicting Lemma 3.4. Finally, note that $E:=\mathcal{S}_{\beta} \backslash\left(\mathcal{S}_{\beta}+T\right)$ is bounded by $\max \left(s_{1}, \ldots, s_{k}\right)+(f+1) e$ and hence finite. Clearly, $\theta$ is determined on $\mathcal{S}_{\beta} \backslash E$ by (3).

\section{Injective Rota-Baxter operators on $k[x]$}

For now let $\boldsymbol{k}$ be an arbitrary field of characteristic zero. An important subclass of Rota-Baxter operators $P$ on $\boldsymbol{k}[x]$ are those associated with the standard derivation $\partial$ in the sense that $\partial \circ P=1_{\boldsymbol{k}}[x]$. We generalize this for arbitrary $r \in \boldsymbol{k}[x]^{\times}$to the differential law $\partial \circ P=r$, where $r$ denotes the corresponding multiplication operator. Thus we define

$$
\operatorname{RBO}_{r}(\boldsymbol{k}[x]):=\{P \in \operatorname{RBO}(\boldsymbol{k}[x]) \mid \partial \circ P=r\} .
$$

Let us now show that the class of all operators satisfying a differential law actually coincides with the class of all injective operators, which we denote by $\mathrm{RBO}_{*}(\boldsymbol{k}[x])$. 


\section{Theorem 4.1.}

$$
\mathrm{RBO}_{*}(\boldsymbol{k}[x])=\bigcup_{r \in \boldsymbol{k}[x]^{\times}} \mathrm{RBO}_{r}(\boldsymbol{k}[x]) .
$$

Proof. The inclusion from right to left is simple as $P(f)=0$ implies $\partial(P(f))=$ $r f=0$ and hence $f=0$ since $\boldsymbol{k}[x]$ is an integral domain.

Now let $P: \boldsymbol{k}[x] \rightarrow \boldsymbol{k}[x]$ be an injective Rota-Baxter operator. Then there exists a linear map $D: \operatorname{im} P \rightarrow \boldsymbol{k}[x]$ with $D \circ P=1_{\boldsymbol{k}[x]}$. Adjoining $\boldsymbol{k}$ as constants, one can immediately check that $D$ is a derivation on the unitary subalgebra $J:=\boldsymbol{k}+\operatorname{im} P$. Note that $P(1) \notin \boldsymbol{k}$ since $P(1)=c$ implies $c^{2}=P(1)^{2}=2 P(P(1))=2 c^{2}$ and hence $c=0$, contradicting injectivity. This means $\boldsymbol{k} \subsetneq J$. Since $J \subseteq \boldsymbol{k}[x]$ is an integral domain, $D$ extends uniquely to a derivation on the fraction field $K \subseteq \boldsymbol{k}(x)$ of the ring $J$. By Lüroth's theorem [Cohn 2003, Theorem 11.3.4], the intermediate field $\boldsymbol{k} \subset K \subseteq \boldsymbol{k}(x)$ is a simple transcendental extension of $\boldsymbol{k}$, so there exists $\phi \in \boldsymbol{k}(x) \backslash \boldsymbol{k}$ with $K=\boldsymbol{k}(\phi)$. But then $K \subseteq \boldsymbol{k}(x)$ is an algebraic field extension [van der Waerden $1993, \S 73$ ], so the derivation $D$ extends uniquely to $\boldsymbol{k}(x)$ according to [Cohn 2003, Theorem 11.5.3]. But it is well known [Nowicki 1994, Proposition 1.3.2] that every $\boldsymbol{k}$-derivation on $\boldsymbol{k}[x]$ is a multiple of the canonical derivation, so we must have $D=\psi \partial$ for $\psi:=D(x)$. Then $D \circ P=1$ on $\boldsymbol{k}[x]$ implies that $\psi \cdot P(1)^{\prime}=1$, so we obtain $D=r^{-1} \partial$ with $r:=P(1)^{\prime} \in \boldsymbol{k}[x]$ and then also $\partial \circ P=r$.

Thus the study of injective Rota-Baxter operators on $\boldsymbol{k}[x]$ reduces to the study of $\mathrm{RBO}_{r}(\boldsymbol{k}[x])$. As noted in Section 2, all standard integral operators $J_{a}$ are in $\mathrm{RBO}_{1}(\boldsymbol{k}[x])$; more generally, the analytically modeled operators $J_{a} r$ are in $\mathrm{RBO}_{r}(\boldsymbol{k}[x])$. It is thus tempting to speculate that $\mathrm{RBO}_{r}(\boldsymbol{k}[x])$ is exhausted by the $J_{a} r$. For the special case $\boldsymbol{k}=\mathbb{R}$ and $r=x^{k}$ this will be proved at the end of this section in Theorem 4.9. For the moment, let $\boldsymbol{k}$ be an arbitrary field containing $\mathbb{Q}$.

From integration over the reals, it is well known that the difference between two indefinite integrals is always a definite integral, which may be interpreted as a measure. This generalizes to the algebraic setting in the following way:

Lemma 4.2. Let $r \in \boldsymbol{k}[x]^{\times}$and $a \in \boldsymbol{k}$ be arbitrary. Then $P \in \operatorname{End}(\boldsymbol{k}[x])$ satisfies the differential law $\partial \circ P=r$ if and only if $J_{a} r-P \in \boldsymbol{k}[x]^{*}$.

Proof. Since $\partial \circ J_{a} r=r$, a linear operator $P$ on $\boldsymbol{k}[x]$ satisfies $\partial \circ P=r$ if and only if $\partial \circ \mu=0$ for $\mu:=J_{a} r-P$. The latter identity holds if and only if im $\mu$ is contained in $\operatorname{ker} \partial=\boldsymbol{k}$.

In analogy to the reals, we call the above linear functional $\mu$ the associated measure of $P$. Then the lemma says that the linear operators satisfying the differential law are classified by their associated measures in the sense that

$$
\{P \in \operatorname{End}(\boldsymbol{k}[x]) \mid \partial \circ P=r\}=J_{a} r+\boldsymbol{k}[x]^{*},
$$

where the initialized point $a$ may be chosen arbitrarily (typically $a=0$ ). But in the real case, a measure is more than an arbitrary linear functional; for the algebraic 
situation this is captured in the following result. Here and henceforth we employ the abbreviation $\star_{r, a}$ for $\star_{J_{a} r}$, and $\star_{r}$ for $\star_{r, 0}$.

Theorem 4.3. Let $r \in \boldsymbol{k}[x]^{\times}$and $a \in \boldsymbol{k}$ be arbitrary. Then the map defined by $P \mapsto J_{a} r-P$ is a bijection between $\mathrm{RBO}_{r}(\boldsymbol{k}[x])$ and $\left(\boldsymbol{k}[x], \star_{r, a}\right)^{\bullet}$.

Proof. By Lemma 4.2 and Proposition 2.3(c), we obtain an surjective map

$$
\mathrm{RBO}_{r}(\boldsymbol{k}[x]) \rightarrow\left(\boldsymbol{k}[x], \star_{r, a}\right)^{\bullet}, \quad P \mapsto J_{a} r-P .
$$

The map is injective since $J_{a} r-P=J_{a} r-\widetilde{P}$ implies $P=\widetilde{P}$.

Thus the preceding classification of operators satisfying a differential law may be refined to

$$
\mathrm{RBO}_{r}(\boldsymbol{k}[x])=J_{a} r-\left(\boldsymbol{k}[x], \star_{r, a}\right)^{\bullet} .
$$

For working out a more explicit description, we specialize to the monomial case $r=x^{k}$, where we use the abbreviation $\star_{k}$ for $\star_{x^{k}}$. To this end, we will determine $\left(\boldsymbol{k}[x], \star_{k}\right)^{\bullet}$, starting with $k=0$.

Theorem 4.4. (a) For any $k \in \mathbb{N}$, the isomorphism $\left(\boldsymbol{k}[x], \star_{k}\right) \cong x^{k+1} \boldsymbol{k}[x]$ of nonunitary algebras holds.

(b) There is a bijection $\left(\boldsymbol{k}[x], \star_{0}\right)^{\bullet} \rightarrow \boldsymbol{k}$ that sends $\mu$ to $\mu(1)$. In particular, the value $a:=\mu(1) \in \boldsymbol{k}$ determines $\mu$ uniquely by

$$
\mu\left(x^{n}\right)=\frac{1}{n+1} a^{n+1}
$$

for all $n \in \mathbb{N}$. Moreover, the codimension of $\operatorname{ker} \mu$ equals 0 for $a=0$, and 1 for $a \neq 0$.

Proof. (a) Note that $\left\{u_{n}:=n x^{n-k-1} \mid n \geqslant k+1\right\}$ is a $\boldsymbol{k}$-linear basis of $\boldsymbol{k}[x]$ with

$$
\begin{aligned}
u_{m} \star_{k} u_{n} & =m x^{m-k-1} J_{0}\left(x^{k} \cdot n x^{n-k-1}\right)+n x^{n-k-1} J_{0}\left(x^{k} \cdot m x^{m-k-1}\right) \\
& =m x^{m-k-1} x^{n}+n x^{n-k-1} x^{m}=(m+n) x^{m+n-k-1}=u_{m+n} .
\end{aligned}
$$

Thus the $\boldsymbol{k}$-linear map induced by $u_{n} \mapsto x^{n}(n \geqslant k+1)$ is an isomorphism $\left(\boldsymbol{k}[x], \star_{k}\right) \rightarrow x^{k+1} \boldsymbol{k}[x]$ of nonunitary $\boldsymbol{k}$-algebras as claimed.

(b) Since $x \boldsymbol{k}[x]$ is the free nonunitary commutative $\boldsymbol{k}$-algebra on $x$, so is $\left(\boldsymbol{k}[x], \star_{0}\right)$ by the isomorphism from (a). Then the bijection follows from the universal property of free nonunitary commutative $\boldsymbol{k}$-algebra on $x$. Note that under the isomorphism from (a), the generator $x$ of $x \boldsymbol{k}[x]$ corresponds to the generator $1=u_{1}$ of $\left(\boldsymbol{k}[x], \star_{0}\right)$.

To prove (18), we use induction on $n$. For the base case $n=0$, we get $\mu(1)=a$ by the definition of $a$. Now suppose (18) has been proved for a fixed $n$. Since

$$
1 \star_{0} x^{n}=J_{0}\left(x^{n}\right)+x^{n} J_{0}(1)=\frac{n+2}{n+1} x^{n+1}
$$


and $\mu$ is a $\boldsymbol{k}$-algebra homomorphism, we have

$$
\mu\left(\frac{n+2}{n+1} x^{n+1}\right)=\mu\left(1 \star_{0} x^{n}\right)=\mu(1) \mu\left(x^{n}\right)=\frac{1}{n+1} a^{n+2},
$$

applying the induction hypothesis in the last step. Thus we obtain $\mu\left(x^{n+1}\right)=$ $(1 /(n+2)) a^{n+2}$, and the induction is complete. The last statement follows since the codimension of ker $\mu$ equals the dimension of $\operatorname{im} \mu$ and $\mu$ is surjective if and only if $\mu(1) \neq 0$.

At this juncture, the results accumulated are sufficient for classifying all RotaBaxter operators $P$ satisfying the differential relation $\partial \circ P=1_{\boldsymbol{k}}[x]$. This is an important special case since it states that all indefinite integrals are analytically modeled.

Theorem 4.5. We have $\mathrm{RBO}_{1}(\boldsymbol{k}[x])=\left\{J_{a} \mid a \in \boldsymbol{k}\right\}$.

Proof. The inclusion from right to left is clear, so assume $P \in \mathrm{RBO}_{1}(\boldsymbol{k}[x])$. By Theorem 4.3 , there exists $\mu \in\left(\boldsymbol{k}[x], \star_{0}\right)^{\bullet}$ such that $P=J_{0}-\mu$. Setting now $a:=\mu(1)$, Theorem 4.4 asserts that $\mu\left(x^{n}\right)=(1 /(n+1)) a^{n+1}$ for all $n \in \mathbb{N}$. Then

$$
P\left(x^{n}\right)=J_{0}\left(x^{n}\right)-\mu\left(x^{n}\right)=\frac{x^{n+1}-a^{n+1}}{n+1}=J_{a}\left(x^{n}\right),
$$

so that $P=J_{a}$; and the inclusion from left to right is established.

For classifying the Rota-Baxter operators $P$ with $\partial \circ P=x^{k}(k>0)$ we must determine all algebra homomorphisms $\mu$ with respect to the multiplication $\star_{k}$. At this point, we have to restrict ourselves to the field $\boldsymbol{k}=\mathbb{R}$ since we shall make use of the order on the reals in the next two lemmas.

Lemma 4.6. Let $\mu:\left(\mathbb{R}[x], \star_{2 \ell+1}\right) \rightarrow \mathbb{R}$ be an $\mathbb{R}$-algebra homomorphism with $\ell \geqslant 0$. Then we get $\mu(1) \geqslant 0$.

Proof. Since $1 \star_{2 \ell+1} 1=2 J_{0}\left(x^{2 \ell+1}\right)=x^{2 \ell+2} /(\ell+1)$ and $\mu$ is an $\mathbb{R}$-algebra homomorphism, we obtain $c^{2}=\mu(1 \star 2 \ell+11)=\mu\left(x^{2 \ell+2} /(\ell+1)\right)$, where we have set $c:=\mu(1)$. Hence we get the relation $\mu\left(x^{2 \ell+2}\right)=(\ell+1) c^{2}$. We have also

$$
1 \star 2 \ell+1 x^{2 \ell+2}=J_{0}\left(x^{4 \ell+3}\right)+x^{2 \ell+2} J_{0}\left(x^{2 \ell+1}\right)=\frac{3}{4 \ell+4} x^{4 \ell+4},
$$

which implies by the $\mathbb{R}$-algebra homomorphism property and the previous relation that

$$
\mu\left(x^{4 \ell+4}\right)=\frac{4}{3}(\ell+1)^{2} c^{3} .
$$

Next we observe that $x^{\ell+1} \star_{2 \ell+1} x^{\ell+1}=2 x^{\ell+1} J_{0}\left(x^{3 \ell+2}\right)=\frac{2}{3} x^{4 \ell+4} /(\ell+1)$. Setting $\tilde{c}:=\mu\left(x^{\ell+1}\right)$, this yields yet another relation

$$
\mu\left(x^{4 \ell+4}\right)=\frac{3}{2}(\ell+1) \tilde{c}^{2} .
$$


Combining (19) and (20), we obtain $\frac{4}{3}(\ell+1)^{2} c^{3}=\frac{3}{2}(\ell+1) \tilde{c}^{2}$ and thus $c=$ $\sqrt[3]{(9 /(8(\ell+1))) \tilde{c}^{2}} \geqslant 0$.

Lemma 4.7. Let $\mu:\left(\mathbb{R}[x], \star_{k}\right) \rightarrow \mathbb{R}$ be an $\mathbb{R}$-algebra homomorphism for $k \in \mathbb{N}$. Then there exists a number $a \in \mathbb{R}$ such that $\mu(1)=a^{k+1} /(k+1)$.

Proof. We set $c:=\mu(1)$ and $a:=\sqrt[k+1]{(k+1) c}$. If $k=2 \ell+1$ with $\ell \in \mathbb{N}$, Lemma 4.6 implies that $c \geqslant 0$ and we may extract an even root to obtain $a \in \mathbb{R}$. If on the other hand $k=2 \ell$ for $\ell \in \mathbb{N}$, the root in $a$ is odd and hence clearly $a \in \mathbb{R}$ also in this case.

The number $a$ ensured by the previous lemma serves to characterize the associated measure $\mu$ of the Rota-Baxter operator underlying the double product $\star_{k}$. Analytically speaking, $\mu(1)$ is the Riemann integral over $[0, a]$.

Proposition 4.8. Let $\mu:\left(\mathbb{R}[x], \star_{k}\right) \rightarrow \mathbb{R}$ be an $\mathbb{R}$-algebra homomorphism. Then there exists a number $a \in \mathbb{R}$ such that $\mu\left(x^{n}\right)=a^{n+k+1} /(n+k+1)$ for all $n \in \mathbb{N}$. In particular, $\mu$ is uniquely determined by $a$.

Proof. We prove the claim by induction on $n \in \mathbb{N}$. In the base case $n=0$, Lemma 4.7 yields $\mu(1)=a^{k+1} /(k+1)$. Now suppose the claim has been proved up to a fixed $n$. Since

$$
1 \star_{k} x^{n-k}=J_{0}\left(x^{n}\right)+x^{n-k} J_{0}\left(x^{k}\right)=\frac{n+k+2}{(n+1)(k+1)} x^{n+1}
$$

and $\mu$ is an $\mathbb{R}$-algebra homomorphism, we obtain

$$
\begin{aligned}
\mu\left(\frac{n+k+2}{(n+1)(k+1)} x^{n+1}\right) & =\mu\left(1 \star_{k} x^{n-k}\right)=\mu(1) \mu\left(x^{n-k}\right) \\
& =\frac{1}{(n+1)(k+1)} a^{n+k+2}
\end{aligned}
$$

where we have applied the induction hypothesis in the last step since $n-k \leqslant n$. But this gives immediately $\mu\left(x^{n+1}\right)=a^{n+k+2} /(n+k+2)$, which completes the induction.

Since the number $a$ of the proposition above characterizes the associated measures, we obtain now the desired classification of the Rota-Baxter operators $P$ on $\mathbb{R}[x]$ that satisfy the differential relation $\partial \circ P=x^{k}$. The number $a$ plays the role of the initialization point of the integral (we regain the standard integral $J_{0}$ for $a=0$ since then the associated measure is zero).

Theorem 4.9. We have $\mathrm{RBO}_{x^{k}}(\mathbb{R}[x])=\left\{J_{a} x^{k} \mid a \in \mathbb{R}\right\}$ for any $k \in \mathbb{N}$. 
Proof. The inclusion from right to left is clear, so assume $P \in \mathrm{RBO}_{x^{k}}(\mathbb{R}[x])$. Then Theorem 4.3 yields an $\mathbb{R}$-algebra homomorphism $\mu:\left(\mathbb{R}[x], \star_{k}\right) \rightarrow \mathbb{R}$ such that $P=J_{0} x^{k}-\mu$. By Proposition 4.8 , there exists a number $a \in \mathbb{R}$ such that $\mu\left(x^{n}\right)=a^{n+k+1} /(n+k+1)$. Thus

$$
P\left(x^{n}\right)=J_{0}\left(x^{n+k}\right)-\mu\left(x^{n}\right)=\frac{x^{n+k+1}-a^{n+k+1}}{n+k+1}=J_{a}\left(x^{n+k}\right),
$$

so that $P=J_{a} x^{k}$, and the inclusion from left to right is established.

As mentioned earlier, it is tempting to generalize the above result from monomials to arbitrary polynomials. Together with Theorem 4.1, this would imply that:

Conjecture 4.10. $\mathrm{RBO}_{*}(\mathbb{R}[x])=\bigcup_{r \in \mathbb{R}[x]^{\times}} \mathrm{RBO}_{r}(\mathbb{R}[x])=\left\{J_{a} r \mid a \in \mathbb{R}, r \in \mathbb{R}[x]^{\times}\right\}$.

For this we only need to verify

$$
\mathrm{RBO}_{r}(\mathbb{R}[x]) \subseteq\left\{J_{a} r \mid a \in \mathbb{R}\right\} \quad \text { for any } r \in \mathbb{R}[x]^{\times} .
$$

In the rest of this paper, we add some preliminary results in support of this conjecture. Let us call a Rota-Baxter operator $P$ on $\mathbb{R}[x]$ initialized at a point $a \in \mathbb{R}$ if $\mathrm{ev}_{a} \circ P$ is the zero operator, where $\mathrm{ev}_{a}: \mathbb{R}[x] \rightarrow \mathbb{R}[x]$ denotes evaluation at $a$. The typical case is when $P=J_{a} r$. It is easy to see that Conjecture 4.10 is equivalent to the claim that all Rota-Baxter operators in $\mathrm{RBO}_{r}(\mathbb{R}[x])$ are initialized. Indeed, if $P$ is initialized at $a$, then we may multiply the differential law $\partial \circ P=r$ by $J_{a}$ from the left to obtain $P=J_{a} r$ since we have $J_{a} \partial=1_{\mathbb{R}[x]}-\mathrm{ev}_{a}$. So for proving Conjecture 4.10 one has to determine the initialization point $a$ from a given Rota-Baxter operator $P$ and $r \in \mathbb{R}[x]^{\times}$. If $P$ is already known to be of the form $J_{a} r$, this can be done as follows:

Lemma 4.11. For the Rota-Baxter operator $P=J_{a} r$ with $a \in \mathbb{R}$ and $r \in \mathbb{R}[x]^{\times}$, we have

$$
a=\frac{P\left(2 x r^{\prime}+r\right)-x r^{2}}{P\left(2 r^{\prime}\right)-r^{2}}
$$

provided $r(a) \neq 0$. On the other hand, if $r(a)=0$ then $P=\left(r-J_{a} r^{\prime}\right) \circ J_{0}$.

Proof. Let us first consider the generic case $r(a) \neq 0$. Using the differential law $\partial \circ P=r$, one sees immediately that numerator and denominator are both constants since they vanish under $\partial$. Moreover, the denominator cannot be zero since

$$
P\left(2 r^{\prime}\right)=\int_{a}^{x}\left(r^{2}\right)^{\prime}=r^{2}-r(a)^{2} \neq r^{2}
$$


by the assumption of genericity. Integrating $\left(r^{2} r^{(i)}\right)^{\prime}=2 r r^{\prime} r^{(i)}+r^{2} r^{(i+1)}$ from $a$ to $x$, we obtain

$$
r^{2} r^{(i)}-r(a)^{2} r^{(i)}(a)=P\left(2 r^{\prime} r^{(i)}+r r^{(i+1)}\right) .
$$

Assuming $r$ has degree $n$, we can write

$$
r=1+r_{1} x+r_{2} \frac{x^{2}}{2 !}+\cdots+r_{n} \frac{x^{n}}{n !},
$$

so that $r^{(n-1)}=r_{n-1}+r_{n} x$ and $r^{(n)}=r_{n}$. Substituting $i=n-1$ and $r(a)^{2}=$ $r^{2}-P\left(2 r^{\prime}\right)$ in (23), we obtain the relation

$$
\left(r_{n-1}+r_{n} x\right) r^{2}-\left(r^{2}-P\left(2 r^{\prime}\right)\right)\left(r_{n-1}+r_{n} a\right)=P\left(2 r_{n-1} r^{\prime}+2 r_{n} x r^{\prime}+r_{n} r\right)
$$

which simplifies to $(x-a) r^{2}=P\left(2 x r^{\prime}-2 a r^{\prime}+r\right)$. Solving this for $a$ gives (22).

Now assume $r(a)=0$. Then for $f \in \mathbb{R}[x]$ we obtain

$$
P f^{\prime}=\int_{a}^{x} r f^{\prime}=[r f]_{a}^{x}-\int_{a}^{x} r^{\prime} f=r f-J_{a} r^{\prime} f,
$$

and hence by $\left(J_{0} f\right)^{\prime}=f$, the required identity $P f=\left(r J_{0}\right) f-\left(J_{a} r^{\prime} J_{0}\right) f=$ $\left(r-J_{a} r^{\prime}\right) J_{0}(f)$.

Lemma 4.11 suggests the following strategy for proving Conjecture 4.10. Given an arbitrary $P \in \mathrm{RBO}_{r}(\mathbb{R}[x])$, we determine first the denominator of (22). If it vanishes, we try to find $\widetilde{P} \in \mathrm{RBO}_{r^{\prime}}(\mathbb{R}[x])$ with $P=(r-\widetilde{P}) \circ J_{0}$, and we use induction on the degree of $r$ to handle $\widetilde{P}$. In the generic case of nonvanishing denominator, we compute the value of $a$ from (22), and it suffices to prove that $P$ is initialized at $a$. For doing this, the first step would be to ascertain that $r(a)^{2}=r^{2}-P\left(2 r^{\prime}\right)$. This would imply that $P\left(r^{\prime}\right)$ vanishes at $x=a$ and hence also $P\left(2 x r^{\prime}+r\right)$ by (24). Using the Rota-Baxter axiom and the above relations, one can produce polynomials $p$ such that $P(p)$ vanishes at $x=a$. If this is done for sufficiently many polynomials $p$ to generate $\mathbb{R}[x]$ as a real vector space, we are done. Here is an example of a class of polynomials where one can infer vanishing at $x=a$, provided $r(a)^{2}=r^{2}-P\left(2 r^{\prime}\right)$ has been established. For $P=J_{a} r$, it recovers the fact that $J_{a}\left(r^{\prime} r^{2 k+1}\right)=(2 k+2)^{-1} J_{a}\left(\left(r^{2 k+2}\right)^{\prime}\right)=(2 k+2)^{-1}\left(r^{2 k+2}-r(a)^{2 k+2}\right)$.

Lemma 4.12. Let $P \in \mathrm{RBO}_{r}(\mathbb{R}[x])$ be arbitrary. Then we obtain $P\left(r^{\prime} r^{2 k}\right)=$ $(2 k+2)^{-1}\left(r^{2 k+2}-c^{k+1}\right)$ for $c:=r^{2}-P\left(2 r^{\prime}\right) \in \mathbb{R}$ and all $k \geqslant 0$.

Proof. We use induction on $k$. The base case $k=0$ is immediate from the definition of $c$. Now assume the claim for all degrees below a fixed $k>0$; we prove it for $k$. By the Rota-Baxter axiom and the definition of $c$ we find

$$
\begin{aligned}
P\left(r^{\prime}\right)^{k+1} & =(k+1) ! P_{r^{\prime}}^{k+1}(1)=(k+1) ! P\left(r^{\prime} P_{r^{\prime}}^{k}(1)\right)=(k+1) P\left(r^{\prime} P\left(r^{\prime}\right)^{k}\right) \\
& =2^{-k}(k+1) P\left(r^{\prime}\left(r^{2}-c\right)^{k}\right),
\end{aligned}
$$


where $P_{r^{\prime}}: \mathbb{R}[x] \rightarrow \mathbb{R}[x]$ is defined by $P_{r^{\prime}}(p):=P\left(r^{\prime} p\right)$. Substituting the defining relation of $c$ on the left-hand side, we obtain $\left(r^{2}-c\right)^{k+1}=2(k+1) P\left(r^{\prime}\left(r^{2}-c\right)^{k}\right)$, so the binomial theorem yields

$$
(2 k+2) P\left(r^{\prime} r^{2 k}\right)=\left(r^{2}-c\right)^{k+1}-2(k+1) \sum_{l=0}^{k-1}\left(\begin{array}{l}
k \\
l
\end{array}\right)(-c)^{k-l} P\left(r^{\prime} r^{2 l}\right) \text {. }
$$

Applying the induction hypothesis leads to

$$
\begin{aligned}
& (2 k+2) P\left(r^{\prime} r^{2 k}\right) \\
& =\left(r^{2}-c\right)^{k+1}-(k+1) \sum_{l=0}^{k-1}\left(\begin{array}{l}
k \\
l
\end{array}\right) \frac{(-c)^{k-l}}{l+1}\left(\left(r^{2}\right)^{l+1}-c^{l+1}\right) \\
& =\left(r^{2}-c\right)^{k+1}+\left(r^{2 k+2}-c^{k+1}\right)-(k+1) \sum_{l=0}^{k}\left(\begin{array}{l}
k \\
l
\end{array}\right) \frac{(-c)^{k-l}}{l+1}\left(\left(r^{2}\right)^{l+1}-c^{l+1}\right) .
\end{aligned}
$$

For evaluating the above sum, just note that integrating $(x-c)^{k}$ from $\alpha$ to $\beta$ and using the binomial theorem gives

$$
\frac{(\beta-c)^{k+1}-(\alpha-c)^{k+1}}{k+1}=\sum_{l=0}^{k}\left(\begin{array}{l}
k \\
l
\end{array}\right) \frac{(-c)^{k-l}}{l+1}\left(\beta^{l+1}-\alpha^{l+1}\right),
$$

which may be evaluated at $(\alpha, \beta)=\left(c, r^{2}\right)$ in the previous sum to obtain $(2 k+2) P\left(r^{\prime} r^{2 k}\right)=\left(r^{2}-c\right)^{k+1}+r^{2 k+2}-c^{k+1}-\left(r^{2}-c\right)^{k+1}=r^{2 k+2}-c^{k+1}$,

which completes the induction.

We conclude with a simple result about the double product $\star$ in the general case of $J_{a} r$. This lemma is a kind of analogy (though not a generalization) of Theorem 4.4(a). In fact, the two results coincide for $r=x$.

Lemma 4.13. Let $\star$ be the double product corresponding to the Rota-Baxter operator $J_{a} r$ and set $\rho=r(a)$. Then the nonunitary subalgebra of $(\boldsymbol{k}[x], \star)$ generated by $u_{n}=n r^{n-2} r^{\prime}(n \geqslant 2)$ is isomorphic to the nonunitary subalgebra of $(\boldsymbol{k}[x], \cdot)$ generated by $x^{n}-\rho^{n}(n \geqslant 2)$.

Proof. The double product of the basis elements $u_{m}(m \geqslant 2)$ and $u_{n}(n \geqslant 2)$ is given by

$$
\begin{aligned}
u_{m} \star u_{n} & =m n r^{m-2} r^{\prime} J_{a} r^{n-1} r^{\prime}+m n r^{n-2} r^{\prime} J_{a} r^{m-1} r^{\prime} \\
& =m r^{m-2} r^{\prime}\left(r^{n}-\rho^{n}\right)+n r^{n-2} r^{\prime}\left(r^{m}-\rho^{m}\right) \\
& =u_{m+n}-\rho^{n} u_{m}-\rho^{m} u_{n},
\end{aligned}
$$


so the $\boldsymbol{k}$-linear map $\phi$ defined by $\phi\left(u_{m}\right)=x^{m}-\rho^{m}$ is a homomorphism of nonunitary $\boldsymbol{k}$-algebras since

$$
\left(x^{m}-\rho^{m}\right)\left(x^{n}-\rho^{n}\right)=\left(x^{m+n}-\rho^{m+n}\right)-\rho^{n}\left(x^{m}-\rho^{m}\right)-\rho^{m}\left(x^{n}-\rho^{n}\right) .
$$

The map $\phi$ is clearly bijective as it maps a $\boldsymbol{k}$-basis to a $\boldsymbol{k}$-basis.

\section{Acknowledgements}

This work was supported by the National Science Foundation of US (Grant No. DMS1001855), the Engineering and Physical Sciences Research Council of UK (Grant No. EP/I037474/1) and the National Natural Science Foundation of China (Grant No. 11371178). L. Guo and S. Zheng also thank the Kavli Institute for Theoretical Physics China and the Morning Center of Mathematics in Beijing for hospitality and support.

\section{References}

[Aguiar 2001] M. Aguiar, "On the associative analog of Lie bialgebras", J. Algebra 244:2 (2001), 492-532. MR 2003c:17035 Zbl 0991.16033

[Atkinson 1963] F. V. Atkinson, "Some aspects of Baxter's functional equation”, J. Math. Anal. Appl. 7 (1963), 1-30. MR 27 \#5135 Zbl 0118.12903

[Bai 2007] C. Bai, "A unified algebraic approach to the classical Yang-Baxter equation", J. Phys. A 40:36 (2007), 11073-11082. MR 2009b:17014 Zbl 1118.17008

[Bai et al. 2010] C. Bai, L. Guo, and X. Ni, "Nonabelian generalized Lax pairs, the classical YangBaxter equation and PostLie algebras”, Comm. Math. Phys. 297:2 (2010), 553-596. MR 2011i:17032 Zbl 1206.17020

[Bai et al. 2013] C. Bai, O. Bellier, L. Guo, and X. Ni, "Splitting of operations, Manin products, and Rota-Baxter operators”, Int. Math. Res. Not. 2013:3 (2013), 485-524. MR 3021790

[Baxter 1960] G. Baxter, "An analytic problem whose solution follows from a simple algebraic identity”, Pacific J. Math. 10 (1960), 731-742. MR 22 \#9990 Zbl 0095.12705

[Birkhoff 1950] G. Birkhoff, "Moyennes des fonctions bornées”, pp. 143-153 in Algèbre et Théorie des Nombres, Colloques Internationaux du Centre National de la Recherche Scientifique 24, Centre National de la Recherche Scientifique, Paris, 1950. MR 13,361d Zbl 0041.44306

[Cartier 1972] P. Cartier, "On the structure of free Baxter algebras", Advances in Math. 9 (1972), 253-265. MR 49 \#2807 Zbl 0267.60052

[Cohn 2003] P. M. Cohn, Basic algebra: Groups, rings and fields, Springer, London, 2003. MR 2003m: 00001 Zbl 1003.00001

[Connes and Kreimer 2000] A. Connes and D. Kreimer, "Renormalization in quantum field theory and the Riemann-Hilbert problem, I: The Hopf algebra structure of graphs and the main theorem", Comm. Math. Phys. 210:1 (2000), 249-273. MR 2002f:81070 Zbl 1032.81026

[Ebrahimi-Fard et al. 2004] K. Ebrahimi-Fard, L. Guo, and D. Kreimer, "Spitzer's identity and the algebraic Birkhoff decomposition in pQFT”, J. Phys. A 37:45 (2004), 11037-11052. MR 2006b:81332 Zbl 1062.81113 
[Ebrahimi-Fard et al. 2006] K. Ebrahimi-Fard, L. Guo, and D. Manchon, "Birkhoff type decompositions and the Baker-Campbell-Hausdorff recursion", Comm. Math. Phys. 267:3 (2006), 821-845. MR 2008c:17020 Zbl 1188.17020

[Gao et al. 2014] X. Gao, L. Guo, and S. Zheng, "Construction of free commutative integrodifferential algebras by the method of Gröbner-Shirshov bases”, J. Algebra Appl. 13:5 (2014), 1350160. MR 3190089 Zbl 06303780

[Guo 2009] L. Guo, "What is. . a Rota-Baxter algebra?", Notices Amer. Math. Soc. 56:11 (2009), 1436-1437. MR 2590727 Zbl 1184.16038

[Guo 2012] L. Guo, An introduction to Rota-Baxter algebra, Surveys of Modern Mathematics 4, International Press, Somerville, MA, 2012. MR 3025028 Zbl 1271.16001

[Guo and Keigher 2000] L. Guo and W. Keigher, "Baxter algebras and shuffle products", Adv. Math. 150:1 (2000), 117-149. MR 2001g:05015 Zbl 0947.16013

[Guo and Keigher 2008] L. Guo and W. Keigher, "On differential Rota-Baxter algebras", J. Pure Appl. Algebra 212:3 (2008), 522-540. MR 2008i:16037 Zbl 1185.16038

[Guo and Zhang 2008] L. Guo and B. Zhang, "Renormalization of multiple zeta values", J. Algebra 319:9 (2008), 3770-3809. MR 2009b:11155 Zbl 1165.11071

[Guo et al. 2014] L. Guo, G. Regensburger, and M. Rosenkranz, "On integro-differential algebras”, J. Pure Appl. Algebra 218:3 (2014), 456-473. MR 3124211 Zbl 06244927

[Nowicki 1994] A. Nowicki, Polynomial derivations and their rings of constants, Uniwersytet Mikołaja Kopernika, Toruń, 1994. MR 2553232 Zbl 1236.13023

[Pei and Guo 2014] J. Pei and L. Guo, "Averaging algebra, Schröder numbers and rooted trees", J. Algebraic Combinatorics (December 2014).

[Reynolds 1895] O. Reynolds, "On the dynamical theory of incompressible viscous fluids and the determination of the criterion”, Phil. Trans. Roy. Soc. A 186 (1895), 123-164. JFM 26.0872.02

[Rosales and García-Sánchez 1999] J. C. Rosales and P. A. García-Sánchez, Finitely generated commutative monoids, Nova Science Publishers, Commack, NY, 1999. MR 2000d:20074 Zbl 0966.20028

[Rosenkranz and Regensburger 2008] M. Rosenkranz and G. Regensburger, "Solving and factoring boundary problems for linear ordinary differential equations in differential algebras", J. Symbolic Comput. 43:8 (2008), 515-544. MR 2009d:34067 Zbl 1151.34008

[Rota 1969a] G.-C. Rota, "Baxter algebras and combinatorial identities, I", Bull. Amer. Math. Soc. 75 (1969), 325-329. MR 39 \#5387 Zbl 0192.33801

[Rota 1969b] G.-C. Rota, "Baxter algebras and combinatorial identities, II", Bull. Amer. Math. Soc. 75 (1969), 330-334. MR 39 \#5387 Zbl 0319.05008

[Rota 1995] G.-C. Rota, Gian-Carlo Rota on combinatorics: Introductory papers and commentaries, edited by J. P. S. Kung, Contemporary Mathematicians, Birkhäuser, Boston, MA, 1995. MR 99b:01027 Zbl 0841.01031

[Semenov-Tian-Shansky 1983] M. A. Semenov-Tian-Shansky, "What is a classical $r$-matrix?", Funct. Ana. Appl. 17:4 (1983), 259-272. Zbl 0535.58031

[Spitzer 1956] F. Spitzer, "A combinatorial lemma and its application to probability theory", Trans. Amer. Math. Soc. 82 (1956), 323-339. MR 18,156e Zbl 0071.13003

[van der Waerden 1993] L. B. van der Waerden, Algebra, I, 9th ed., Springer, Berlin, 1993. In German. Zbl 0781.12002

Received May 30, 2014. 
SHANGHUA ZHENG

DEPARTMENT OF MATHEMATICS

JIANGXI NORMAL UNIVERSITY

NANCHANGE, JIANGXI 330022

CHINA

zheng2712801@163.com

LI GUO

DEPARTMENT OF MATHEMATICS AND COMPUTER SCIENCE

RUTGERS UNIVERSITY AT NEWARK

216 SMITH HALL

101 WARREN STREET

NEWARK, NJ 07102

UNITED STATES

liguo@rutgers.edu

MARKUS ROSENKRANZ

SCHOOL OF MATHEMATICS

Statistics AND ACtUARIAL SCIENCE

UNIVERSITY OF KENT

CANTERbury

CT2 7NF

UNITED KINGDOM

M.Rosenkranz@kent.ac.uk 


\title{
PACIFIC JOURNAL OF MATHEMATICS
}

\author{
msp.org/pjm
}

Founded in 1951 by E. F. Beckenbach (1906-1982) and F. Wolf (1904-1989)

\section{EDITORS}

Don Blasius (Managing Editor)

Department of Mathematics

University of California

Los Angeles, CA 90095-1555

blasius@math.ucla.edu

\author{
Paul Balmer \\ Department of Mathematics \\ University of California \\ Los Angeles, CA 90095-1555 \\ balmer@math.ucla.edu \\ Robert Finn \\ Department of Mathematics \\ Stanford University \\ Stanford, CA 94305-2125 \\ finn@math.stanford.edu \\ Sorin Popa \\ Department of Mathematics \\ University of California \\ Los Angeles, CA 90095-1555 \\ popa@math.ucla.edu
}

\author{
Vyjayanthi Chari \\ Department of Mathematics \\ University of California \\ Riverside, CA 92521-0135 \\ chari@math.ucr.edu \\ Kefeng Liu \\ Department of Mathematics \\ University of California \\ Los Angeles, CA 90095-1555 \\ liu@math.ucla.edu \\ Jie Qing \\ Department of Mathematics \\ University of California \\ Santa Cruz, CA 95064 \\ qing@ cats.ucsc.edu
}

\section{PRODUCTION}

Silvio Levy, Scientific Editor, production@msp.org

\section{SUPPORTING INSTITUTIONS}

ACADEMIA SINICA, TAIPEI

CALIFORNIA INST. OF TECHNOLOGY

INST. DE MATEMÁTICA PURA E APLICADA

KEIO UNIVERSITY

MATH. SCIENCES RESEARCH INSTITUTE

NEW MEXICO STATE UNIV.

OREGON STATE UNIV.

\author{
STANFORD UNIVERSITY \\ UNIV. OF BRITISH COLUMBIA \\ UNIV. OF CALIFORNIA, BERKELEY \\ UNIV. OF CALIFORNIA, DAVIS \\ UNIV. OF CALIFORNIA, LOS ANGELES \\ UNIV. OF CALIFORNIA, RIVERSIDE \\ UNIV. OF CALIFORNIA, SAN DIEGO \\ UNIV. OF CALIF., SANTA BARBARA
}

\author{
Daryl Cooper \\ Department of Mathematics \\ University of California \\ Santa Barbara, CA 93106-3080 \\ cooper@math.ucsb.edu \\ Jiang-Hua Lu \\ Department of Mathematics \\ The University of Hong Kong \\ Pokfulam Rd., Hong Kong \\ jhlu@maths.hku.hk \\ Paul Yang \\ Department of Mathematics \\ Princeton University \\ Princeton NJ 08544-1000 \\ yang@math.princeton.edu
}

These supporting institutions contribute to the cost of publication of this Journal, but they are not owners or publishers and have no responsibility for its contents or policies.

See inside back cover or msp.org/pjm for submission instructions.

The subscription price for 2015 is US \$420/year for the electronic version, and \$570/year for print and electronic.

Subscriptions, requests for back issues and changes of subscribers address should be sent to Pacific Journal of Mathematics, P.O. Box 4163, Berkeley, CA 94704-0163, U.S.A. The Pacific Journal of Mathematics is indexed by Mathematical Reviews, Zentralblatt MATH, PASCAL CNRS Index, Referativnyi Zhurnal, Current Mathematical Publications and Web of Knowledge (Science Citation Index).

The Pacific Journal of Mathematics (ISSN 0030-8730) at the University of California, c/o Department of Mathematics, 798 Evans Hall \#3840, Berkeley, CA 94720-3840, is published twelve times a year. Periodical rate postage paid at Berkeley, CA 94704, and additional mailing offices. POSTMASTER: send address changes to Pacific Journal of Mathematics, P.O. Box 4163, Berkeley, CA 94704-0163.

PJM peer review and production are managed by EditFLOW ${ }^{\circledR}$ from Mathematical Sciences Publishers.

\section{PUBLISHED BY}

\section{mathematical sciences publishers \\ nonprofit scientific publishing}

http://msp.org/

(C) 2015 Mathematical Sciences Publishers 


\section{PACIFIC JOURNAL OF MATHEMATICS}

Volume $275 \quad$ No. $2 \quad$ June 2015

A combinatorial characterization of tight fusion frames

MARCIN BOWNIK, KURT LUOTO and EDWARD RICHMOND

Combinatorics of finite abelian groups and Weil representations

295

KUnAL DUTTA and AMritANSHU PRASAD

Compact anti-de Sitter 3-manifolds and folded hyperbolic structures on

325 surfaces

FRANÇOIS GUÉRITAUd, FANNY KASSEL and MAXIME WOLFF

Circular handle decompositions of free genus one knots

361

FABIOLA MANJARREZ-GUTIÉRREZ, VÍCTOR NÚÑEZ and

ENRIQUE RAMÍREZ-LOSADA

A pointwise a-priori estimate for the $\bar{\partial}$-Neumann problem on weakly 409 pseudoconvex domains

R. MichaEL RANGE

Explicit Hilbert-Kunz functions of $2 \times 2$ determinantal rings

MARCUS ROBINSON and IRENA SWANSON

The Johnson-Morita theory for the ring of Fricke characters of free groups

\section{TAKAO SATOH}

Global representations of the conformal group and eigenspaces of the Yamabe operator on $S^{1} \times S^{n}$

MARK R. SEPANSKI and Jose A. Franco

Rota-Baxter operators on the polynomial algebra, integration, and averaging operators

Shanghua Zheng, Li GuO and MARKus Rosenkranz

Correction to the article Quiver grassmannians, quiver varieties and the preprojective algebra

Alistair Savage and Peter Tingley 